\title{
Fuzzy Multilevel Image Thresholding Based on Modified Quick Artificial Bee Colony Algorithm and Local Information Aggregation
}

\author{
Linguo Li, ${ }^{1,2}$ Lijuan Sun, ${ }^{1,3}$ Jian Guo, ${ }^{1,3}$ Chong Han, ${ }^{1,3}$ and Shujing Li ${ }^{2}$ \\ ${ }^{1}$ School of Computer, Nanjing University of Posts and Telecommunications, Nanjing 210003, China \\ ${ }^{2}$ College of Information Engineering, Fuyang Normal University, Fuyang 236041, China \\ ${ }^{3}$ Jiangsu High Technology Research Key Laboratory for Wireless Sensor Networks, Nanjing University of Posts and \\ Telecommunications, Nanjing 210003, China
}

Correspondence should be addressed to Linguo Li; 1lg-1212@163.com

Received 27 July 2016; Revised 26 November 2016; Accepted 28 November 2016

Academic Editor: Erik Cuevas

Copyright (C) 2016 Linguo Li et al. This is an open access article distributed under the Creative Commons Attribution License, which permits unrestricted use, distribution, and reproduction in any medium, provided the original work is properly cited.

\begin{abstract}
Thresholding segmentation based on fuzzy entropy and intelligent optimization is one of the most commonly used and direct methods. This paper takes fuzzy Kapur's entropy as the best optimal objective function, with modified quick artificial bee colony algorithm (MQABC) as the tool, performs fuzzy membership initialization operations through Pseudo Trapezoid-Shaped (PTS) membership function, and finally, according to the image's spacial location information, conducts local information aggregation by way of median, average, and iterative average so as to achieve the final segmentation. The experimental results show that the proposed FMQABC (fuzzy based modified quick artificial bee colony algorithm) and FMQABCA (fuzzy based modified quick artificial bee colony and aggregation algorithm) can search out the best optimal threshold very effectively, precisely, and speedily and in particular show exciting efficiency in running time. This paper experimentally compares the proposed method with Kapur's entropy-based Electromagnetism Optimization (EMO) method, standard ABC, and FDE (fuzzy entropy based differential evolution algorithm), respectively, and concludes that MQABCA is far more superior to the rest in terms of segmentation quality, iterations to convergence, and running time.
\end{abstract}

\section{Introduction}

Thresholding is one of the most direct, simple, and efficient approaches to image segmentation, which can discriminate objects from the background through a set of thresholds at pixel levels. The automatic separation between objects and background remains the most difficult yet intriguing domain for image processing and pattern recognition [1]. It not only can show well defined regions with the minimum overlap and effectiveness of aggregation, but also can provide initial prediction or preprocessing for more complicated segmentation methods (such as level-sets or active contours) [2]. Consequently, thresholding segmentation is regarded as the key link in image analysis and image understanding and therefore is widely used in many areas like medical analysis [3], image classification [4], object recognition [5] and image copy detection [6], and so forth.
Thresholding segmentation is the most commonly used method for image segmentation [7]. Bilevel thresholds are mainly used to separate the object from the background, namely, bilevel thresholding. Multilevel thresholding (MT) can classify the pixels into different groups; hence multiple regions are created, which can lead to finer segmentation results. The thresholding methods proposed in the literature can be divided into two categories, parametric and nonparametric [8-10]. The parametric are usually time consuming and computationally expensive, whereas the nonparametric are more robust and precise and as a result get more attention, as they can determine the optimal threshold by optimizing some standards like between-class variance, the entropy, the error rate, and so forth [8-11]. This paper from the perspective of nonparametric methods will be focused on the study of MT methods through intelligent optimization and fuzzy theory. 
Of nonparametric methods, gray level global thresholding adopting information entropy is the most popular one. Such method takes the intelligent optimization as the tool and by optimizing some information entropy to get the maximum (or minimum) objective function to achieve the final segmentation. The most commonly used objective functions include the maximization of the entropy (e.g., Kapur's entropy) [12], maximization of the between-class variance (e.g., Otsu's method) [13], the use of the fuzzy similarity measure [14], and minimization of the Bayesian error [15]. All of these techniques have been extended into the area of multilevel image segmentation. But the computational complexity for the application of such methods in MT grows dramatically. As a consequence, scholars in recent years have extensively used evolutionary and swarm-based intelligent computational techniques to promote multilevel computational efficiency for multilevel image segmentation and precision of threshold [11]. But the global multilevel thresholds generally get the final segmentation results by maximizing the separation between classes. Yet it neglects the complexity in uneven illumination and soft transitions between gray levels and fails to consider the spatial relationships between pixels, which lead to the imperfections of segmentation results [1]. The biggest problem with the global thresholding lies in the fact that it is pixel oriented rather than region oriented. Therefore, pixels that have the same gray levels are segmented into the same regions if such factors as connectivity of pixels and closeness of objects are not considered. As a result, such methods are prone to create isolated pixels.

Of all the research findings for the various thresholding methods, each one of them is designed specifically to deal with one particular kind of images. Sezgin and Sankur [16] were the first to classify the image thresholding methods into six categories. Aja-Fernández et al. [1] further grouped them into three kinds based on the six classifications. (a) Methods that calculate a global threshold for the whole image. (b) Methods that use an adaptive local threshold. (c) Methods that use spatial local information for classifying the pixels. The first kind was the earliest to be employed. But due to the drawbacks inherent in it, currently, scholars have directed their focus on the latter two. The second kind is an improvement on and complement to the first kind which selects the threshold locally through adaptability so as to avoid local nonadaptability, but the computational complexity will grow accordingly. The third kind is mainly employed to make up for the deficiency in spatial relationship and introduces the local spatial information to heighten the effects of segmented results. In this paper, we introduce local spatial information to solve segmentation problems and at the same time explore the applications of fuzzy logic and fuzzy theory in image thresholding. The starting point of introducing local spatial information comes from the definition of one class or target, where the membership of a pixel is highly correlated with the membership of the pixels of surrounding regions [1]. In order to promote the quality of image segmentation, fuzzy membership function is used to assign each pixel with fuzzy degrees. And according to fuzzy theory, the traditional hard assigning regions or classes will be replaced with soft assigning pattern. Detailedly, the fuzzy Kapur's entropy which is modified by Kapur's entropy is adopted in this method as the objective function. It combines with the MQABC algorithm to generate the optimal thresholds which are used as the centroids for fuzzy membership assignment and local information aggregation.

The rest of the paper is organized as follows. Section 2 introduces related work of fuzzy entropy, intelligent optimization, and local information aggregation in the field of MT. In Section 3, the nonfuzzy hard thresholding and fuzzy soft thresholding are formulated, and then the corresponding Kapur's entropy for image segmentation and the ultimate objective function are presented. In Section 4, the standard bee colony algorithm is briefly described and the proposed MQABC is dealt with in detail. Section 5 analyzes the multiple thresholds-based fuzzy membership initialization and fuzzy aggregation methods. The experiment results and discussions are presented in Section 6. The last section presents the conclusions.

\section{Related Works}

Fuzzy theory and fuzzy logic are well-known to be flexible tools where imprecise knowledge or not-well defined features have to be used [17]. It has been extensively used in system control domain and applied in image processing to some extent [18-21]. In the past 10 years, thresholding segmentation approaches have been proposed based on fuzzy logic and fuzzy calculations. In such approaches, fuzzy logic is combined with clustering method [22], fuzzy compactness [23], the interpretation of thresholds as type 2 fuzzy logic [24], and the soft calculations (such as ant colonies, bee colonies, and bacteria migration heuristics) [11], respectively, mainly used to search for the optimal thresholds, but they failed to take the pixel spatial relationship segmentation methods into full account. In these fuzzy based thresholding methods, clustering methods are among the most popular, but they are highly complicated where computational time is concerned. With the progress and upgradation of optimization methods, fuzzy optimization in thresholding segmentation gets more and more attention and it has been proved feasible to employ intelligent optimization to solve thresholding problems [10]. A large number of literature show that intelligent optimizations are far superior to traditional methods in processing speed, precision and robustness [5, 10]. Oliva et al. [7] introduced Electromagnetism Optimization into thresholding segmentation and compared it with Kapur's entropy and Otsu's method. The experiments show that Kapur's entropy is more efficient. Ghamisi et al. [25] have fully analyzed the performance in thresholding of such methods as Particle swarm optimization (PSO), Darwinian Particle Swarm Optimization (DPSO), and Fractional-order Darwinian Particle Swarm Optimization (FODPSO). In comparison with Bacteria Foraging algorithm $(\mathrm{BF})$ and genetic algorithms (GA), FOPDSO delivers better performance in overcoming local optimum trap and computational speed. Kurban et al., [10] have conducted a comparative study of the applications of the computational techniques involved in MT exhaustively. According to the statistical analysis of 
the objective value, the swarm-based method is more able to precisely solve segmentation problems. In a further analysis, swarm-based thresholding segmentation mainly takes Otsu's method, between-class variance, Tsallis entropy, and Kapur's entropy as the objective functions; the experimental results show that the optimized processing, by way of artificial bee colony, could obtain better segmentation results. Akay [8] took between-class variance and Kapur's entropy as the objective functions, respectively, and compared artificial bee colony method with the particle swarms one; it shows that Kapur's entropy-based artificial bee colony method shows better performance when the number of the thresholds increases, and the complexity of computational time is also reduced. Bhandari et al. [26] conducted detailed comparative analysis between Kapur's Otsu and Tsallis functions through experiments, which shows that in the remote sensing satellite image segmentation, Kapur's entropy-based artificial bee colony method is far superior.

The above-mentioned optimized methods have made great progress in precision, efficiency, and segmentation quality in solving thresholding problems. But classifying regions by hard thresholding will easily lead to edge fuzziness and even isolated pixels [27]. In more recent years, fuzzy theory based thresholding method has become the focus of study, as the proposed soft thresholding strategy can effectively solve the problem. Zhao et al. [28] were the earliest to define the three-membership functions and then applied them to three-level thresholding problems. Based on that, Tao et al., [29] proposed three-level fuzzy entropy based thresholding methods; the three functions, namely, Ifunction, F-function, and S-function, are defined in detail. The method maximizes global fuzzy entropy through Genetic Algorithm (GA) to get the optimal thresholds. These two methods only show the visual effects, failing to present a qualitative analysis. Pratamasunu et al. [30] assigned fuzzy coefficient to each I-function and then surveyed the function to achieve the automation of thresholding by defining the distance between the fuzzy set and its closest crisp set. Although the method shows a comparative advantage over the Lopes method and Otsu thresholding, the experimental results are only applicable to bilevel thresholds and fail to show the effects of MT. Muppidi et al. [31] used Triangular membership function, Trapezoidal membership function, and Bell-shaped membership function to define three kinds of fuzzy entropies, respectively, and search out the optimal parameters set through GA. Such methods are only compared with Otsu thresholding and failed to give adequate quantitative analysis. Sarkar et al., [27] based on image histogram and fuzzy entropy theory, used Different Evolution method to get the optimal threshold to obtain multilevel thresholding segmentation. Compared with PSO and GA, such method delivers better performance in speed and precision and at the same time the author offers a large number of numerical experimental comparisons and data contrast. Aja-Fernández et al. [1] have come up with the solution scheme for multiregion thresholding segmentation by using fuzzy entropy and aggregation methods. The scheme also provides solutions for histogram and fuzzy entropy. More importantly, the scheme noted the drawbacks of pixel spatial relationship which the thresholding segmentation failed to take into account. Based on this, by local information aggregation, it improves the segmentation results and removes the isolated pixels. In local information aggregation methods, the author presents median aggregation, average aggregation, and iterative average aggregation. The experimental results testified to the effectiveness of these aggregation methods. Taken together, the fuzzy and nonfuzzy multilevel thresholding methods, it can be concluded that the proposed method based on fuzzy entropy and intelligent optimization, in combination with local spatial information aggregation, is a feasible solution scheme for multilevel thresholding segmentation.

Artificial bee colony method is a simple yet highly efficient optimized pattern, and its search iteration is much easier to be implemented compared with other heuristic search methods [32]. More importantly, artificial bee colony employs few predefined control parameters, which reduces the interference of artificiality set parameters. But there still exists some drawbacks in its solution search equation, which is fine at exploration but inferior at exploitation process [26]. In quick artificial bee colony methods [33], an advanced solution equation was proposed. In the process of searching out the optimal equation, the onlooker bee searches only around the best solution of previous iterations to improve exploitation. Therefore, the core is to determine the search scope. The scope, if larger than necessary, will greatly heighten the complexity, otherwise it will be reduced to local optimization. However, different problems call for different distance strategies and patterns; it is also true with MT. This paper will be focused on the study of the multilevel thresholding methods which are based on Kapur's entropy, modified quick artificial bee colony algorithm for image segmentation, and local information aggregation.

\section{Formulation of Multilevel Fuzzy Kapur's Entropy}

MT needs to set a set of thresholds $t_{i}$ and the image can be segmented into different regions based on different gray levels. Let image $I$ is be segmented; in order to achieve the final segmentation, the proposed method assumes that every pixel in the image $I$ will have a degree of membership in each of the $N$ regions. That membership will be modeled using fuzzy membership functions. In what follows, we will give the optimal threshold obtained through fuzzy entropy and artificial bee colony and then assign each pixel with a fuzzy degree in different regions.

3.1. Concept of Kapur's Entropy for Hard Thresholding. Based on the above analyses, the intelligent optimization which takes Kapur's entropy as the objective function gets the better segmentation results. Kapur's method can be easily extended from bilevel thresholding to multilevel thresholding. Entropy-based thresholding takes the gray level histogram as the basis. When the entropy reaches the maximization, the optimal threshold will be distributed normally.

Entropy of a discrete information source can be obtained by the probability distribution $p=p_{i}$, where $p_{i}$ is the probability of the system in possible state $i$ [7]. The probability 
for each gray level $i$ is its relative occurrence frequency. Let the number of gray levels for image $I$ be $h(i)$; the probability distribution can be described as follows:

$$
p_{i}=\frac{h(i)}{\sum_{i=0}^{L-1} h(i)} \quad i=0,1, \ldots, L-1
$$

Kapur's entropy is used to measure the compactness and separability of classes. For MT, Kapur's entropy can be described in

$$
\begin{aligned}
& H_{0}=-\sum_{i=0}^{t_{1}-1} \frac{p_{i}}{\omega_{0}} \ln \frac{p_{i}}{\omega_{0}}, \quad \omega_{0}=\sum_{i=0}^{t_{1}-1} p_{i}, \\
& H_{1}=-\sum_{i=t_{1}}^{t_{2}-1} \frac{p_{i}}{\omega_{1}} \ln \frac{p_{i}}{\omega_{1}}, \quad \omega_{1}=\sum_{i=t_{1}}^{t_{2}-1} p_{i}, \\
& H_{j}=-\sum_{i=t_{j}}^{t_{j+1}-1} \frac{p_{i}}{\omega_{j}} \ln \frac{p_{i}}{\omega_{j}}, \quad \omega_{j}=\sum_{i=t_{j}}^{t_{j+1}-1} p_{i}, \\
& H_{m}=-\sum_{i=t_{m}}^{L-1} \frac{p_{i}}{\omega_{m}} \ln \frac{p_{i}}{\omega_{m}}, \quad \omega_{m}=\sum_{i=t_{m}}^{L-1} p_{i} .
\end{aligned}
$$

Thus, the function of (T) can be obtained by (3), used as a parameter of MQABC's fitness function in Section 4.

$$
f(T)=\sum_{i=0}^{m}-H_{i} \quad T=\left[\begin{array}{llll}
t_{0} & t_{1} & \cdots & t_{m}
\end{array}\right]
$$

where $T$ represents a vector quantity of thresholds and $m$ represents the stop value of the number of thresholds.

\subsection{Concept of Fuzzy Kapur's Entropy for Soft Thresholding.} Let the set $R$ be a collection of elements that can either belong to or not belong to region $r$; according to the fuzzy theory, it can be defined as follows:

$$
R=\left\{\left(i, \mu_{r}(i)\right) \mid i \in I\right\}, \quad 0 \leq \mu_{r}(i) \leq 1, r \in[1, N]
$$

where $u_{r}(i)$ is called membership function, which is used to measure the closeness between pixel $i$ and region $r$. The higher the value is, the bigger the probability that the pixel will be distributed into region $r$ is, and $N$ represents the number of regions.

Option of membership function is the key factor for fuzzy thresholding segmentation. A large number of literature adopts different membership functions to test its effectiveness; trapezoidal membership function is the most commonly used and also the most efficient method [31]. Just as in literature [27], in the case that there are four fuzzy parameters, the curve of the fuzzy degrees can be shown in Figure 1.
The fuzzy trapezoidal membership function with four parameters can be easily extended to the scenario of $n$ ( $n=$ $2 *(m+1))$, which can be formulated as in

$$
\begin{gathered}
\mu_{0}(k)= \begin{cases}1, & k \leq a_{1}, \\
\frac{k-a_{2}}{a_{1}-a_{2}}, & a_{1}<k \leq a_{2}, \\
0, & k>a_{2},\end{cases} \\
\mu_{1}(k)= \begin{cases}0, & k \leq a_{1}, \\
\frac{k-a_{1}}{a_{2}-a_{1}}, & a_{1}<k \leq a_{2}, \\
\frac{k-a_{4}}{a_{3}-a_{4},} & a_{2}<k \leq a_{3}, \\
0, & k>a_{4},\end{cases} \\
\mu_{m}(k)= \begin{cases}\frac{k}{0,}, a_{n-1}, & a_{n-1}<k \leq a_{n}, \\
\frac{a_{n}-a_{n-1}}{1,} & k>a_{n},\end{cases}
\end{gathered}
$$

where $a_{1}, a_{2}, \ldots, a_{n}$ represent the fuzzy parameters of the trapezoidal membership function and $k$ is the gray value of a single pixel. Based on the trapezoidal membership function, the fuzzification of Kapur's entropy in Section 3.1 can be described as in

$$
\begin{aligned}
& H_{0}=- \sum_{i=0}^{L-1} \frac{\mu_{0}(i) * p_{i}}{\omega_{0}} \ln \left(\frac{\mu_{0}(i) * p_{i}}{\omega_{0}}\right), \\
& \omega_{0}=\sum_{i=0}^{L-1}\left(\mu_{0}(i) * p_{i}\right), \\
& H_{1}=-\sum_{i=0}^{L-1} \frac{\mu_{1}(i) * p_{i}}{\omega_{1}} \ln \left(\frac{\mu_{1}(i) * p_{i}}{\omega_{1}}\right), \\
& \omega_{j}=-\sum_{i=0}^{L-1} \frac{\mu_{j}(i) * p_{i}}{\omega_{j}} \ln \left(\frac{\mu_{j}(i) * p_{i}}{\omega_{j}}\right), \\
& H_{m}=-\sum_{i=0}^{L-1} \frac{\mu_{m}(i) * p_{i}}{\omega_{m}} \ln \left(\frac{\left.\mu_{m}(i) * p_{i}\right),}{\omega_{m}}\right), \\
& \omega_{m}=\sum_{i=0}^{L-1}\left(\mu_{m}(i) * p_{i}\right) \\
& H_{i=0}^{L-1},
\end{aligned}
$$


In order to get the specific $N$ parameters in (5), this paper will adopt MQABC to minimize the total Kapur's entropy, as shown in (7). By comparing (2) and (6), we can clearly see that the fuzzy entropy involves more complicated computational procedures. In such a case, it is of necessity to adopt intelligent optimization.

$$
\begin{aligned}
\phi\left(a_{1}, a_{2}, \ldots, a_{n}\right)=f(T)=\sum_{i=0}^{m}-H_{i} & \\
T & =\left[\begin{array}{llll}
t_{0} & t_{1} & \cdots & t_{m}
\end{array}\right] .
\end{aligned}
$$

After $N$ parameters are obtained through the solution, the corresponding thresholds can be represented as in

$$
\begin{gathered}
t_{0}=\frac{a_{1}+a_{2}}{2}, \\
t_{1}=\frac{a_{3}+a_{4}}{2} \\
\vdots \\
t_{m}=\frac{a_{n-1}+a_{n}}{2} .
\end{gathered}
$$

\section{Brief Explanation of Quick Artificial Bee Colony Algorithm}

$\mathrm{ABC}$ algorithms have been widely used as they show high efficiency and better convergence performance and employ fewer control parameters, in particular when the tuning of control parameters is more difficult than the problem itself. In fuzzy MT, the fuzzy entropy involves much more procedures of computations; the algorithm which employs a small number of control parameters is advised to be applied. A brief description of the $\mathrm{ABC}$ and the QABC algorithms will be given in the following subsections.

4.1. Standard Artificial Bee Colony Algorithm. In standard $\mathrm{ABC}$, according to the migration behavior, artificial bees can be classified into three types, namely, employed bees, onlooker bees, and scout bees. Employed bees are related to specific food sources; onlooker bees decide to choose a certain food source by observing the dance of employed bees, while scout bees will search food randomly. The specific bee colony behavior and related simulation can be found in literature [32].

In the initialization phase, all the food sources are initialized by (9). The number of food sources is set by predefined parameters.

$$
t_{j, i}=l_{i}+\operatorname{random}(0,1) *\left(u_{i}-l_{i}\right),
$$

where $t_{j, i}$ is the $i$ th dimensional data of the $j$ th food source. $l_{i}$ and $u_{i}$ stand for the lower limit and upper limit of the parameter $t_{j} . t_{j}$ is the $j$ th food source.

In the employed bee phase, a search is conducted in her memory at a specific speed $\varphi_{j, i}$. The speed (as shown in (10)) determines the change rate of the $t_{j, i}$ food source, which, to a certain degree, affects the convergence speed. If the solution produced by (10) is better than the bee's solution, then the memory is updated by a greedy selection approach.

$$
v_{j, i}=t_{j, i}+\varphi_{j, i} *\left(t_{j, i}-t_{r, i}\right),
$$

where $t_{r}$ represents a randomly selected food source, $i$ is a randomly selected parameter index, and $\varphi_{j, i}$ is a random number within the range $[-1,1]$.

To compare the advantages and disadvantages, the fitness of the solution is produced by (11). The higher fitness value represents a better objective function value, thus maximizing the fitness function which can reach the optimal thresholds.

$$
\text { fit }\left(t_{j}\right)= \begin{cases}\frac{1}{\left(1+f\left(t_{j}\right)\right)}, & f\left(t_{j}\right) \geq 0, \\ 1+\operatorname{abs}\left(f\left(t_{j}\right)\right), & f\left(t_{j}\right)<0,\end{cases}
$$

where $f\left(t_{j}\right)$ can be calculated by (4).

An employed bee shares information about food source fitness with an onlooker bee. The onlooker bee by probability selects one source to investigate. The probability function can be represented by

$$
\operatorname{prob}_{j}=\frac{\text { fit }\left(t_{j}\right)}{\sum_{j=1}^{\mathrm{SN}} \text { fit }\left(t_{j}\right)},
$$

where SN represents the number of food sources.

4.2. Modified Quick Artificial Bee Colony Algorithm (MQABC) for Image Thresholding. In standard ABC, the employed bee and the onlooker bee use (10) to search for food sources. In other words, the employed bee and the onlooker bee adopt the same strategies in the same area to search for a new and better food source. But in real honey bee colonies, the employed bee and the onlooker bee adopt different methods to search for new food sources [33]. Therefore, for onlooker bees, when they search for the best food, it is quite reasonable that the pattern differs from the search method. A brand new modified onlooker bee behavior is adopted in the literature with the optimal fitness food source as the center.

The formulated search for new food source can be represented by

$$
v_{N_{j, i}}^{\text {best }}=t_{N_{j, i}}^{\text {best }}+\varphi_{t, i} *\left(t_{N_{t, i}}^{\text {best }}-t_{r, i}\right),
$$

where $t_{N_{i, i}}^{\text {best }}$ is defined as the optimal solution of all the neighborhood food sources around the present food source and $t_{j}, N_{j}$ represents all the neighborhood food sources including $t_{j}$; the rest of the parameters are the same as the ones in (10). It can be clearly observed that the key is how the neighborhood food source is defined.

From (13), the core of this pattern lies in how the neighboring food source is defined. Karaboga and Gorkemli [33] only give a simple definition of neighboring food sources. With regard to different ways of defining problem solution, it needs to define different measurement for similarity. For MT, every solution corresponds to a digital sequence. In this 
paper, the neighboring food source distance is defined as follows.

$$
d_{j, i}=\frac{\sum_{x=1}^{\mathrm{SN}} t_{x, i}}{\mathrm{SN}} \quad i=1,2, \ldots, \mathrm{SL},
$$

where $d_{j, i}$ is the search scope of the food source $t_{j}$ around the neighborhood. SN stands for the number of the food sources and SL is the dimensions of a certain food source. If the Euclidean distance of a solution to $t_{j}$ is shorter than $d_{j, i}$, then it is regarded as the neighborhood of the present solution $t_{j}$, which is different from the standard ABC algorithm. When an onlooker bee reaches the food source $t_{j}$, firstly it will investigate all the neighborhood food sources, choosing the best food source $t_{N_{j}}^{\text {best }}$, and improving her search by (13). In $N_{j}$, the best food source is defined by

$$
\text { fit }\left(t_{N_{j}}^{\text {best }}\right)=\max \left(\text { fit }\left(t_{N_{j}}^{1}\right) \text {, fit }\left(t_{N_{j}}^{2}\right), \ldots, \text { fit }\left(t_{N_{j}}^{s}\right)\right) .
$$
follows.

The improved $\mathrm{ABC}$ algorithm can be represented as Algorithm 1 (main steps of the MQABC algorithm for image thresholding).

Step 1. Initialization of the population size SN, provide the number of thresholds $M$ and maximum cycle number $\mathrm{CN}$, initialization of the population of source foods:

$$
t_{j, i}=l_{i}+\operatorname{random}(0,1) *\left(u_{i}-l_{i}\right)
$$

Then, evaluate the population via the specified optimization function;

Step 2. While (a termination criterion is not satisfied or Cycle Number $<\mathrm{CN})$ do

Step 3. For $j=1$ to $\mathrm{SN}$ do

Produce new solutions (food source positions) $v_{j}$ in the neighborhood of $t_{j}$ for the employed bees using the formula:

$$
v_{j, i}=t_{j, i}+\varphi_{j, i} *\left(t_{j, i}-t_{r, i}\right)
$$

Step 4. Apply the greedy selection process between the corresponding $t_{j}$ and $v_{j}$;

Step 5. Calculate the probability values prob $_{j}$ for the solutions $t_{j}$ by means of their fitness values using the formula:

$$
\operatorname{prob}_{j}=\frac{\text { fit }\left(t_{j}\right)}{\sum_{i=1}^{\mathrm{SN}} \mathrm{fit}\left(t_{j}\right)}
$$

Step 6. Calculate the Euclidean distance $d_{j}$, chose the best food source $t_{N_{j}}^{\text {best }}$ in $N_{j}$;

$$
\begin{aligned}
d_{j, i} & =\frac{\sum_{x=1}^{\mathrm{SN}} t_{x, i}}{\mathrm{SN}} \quad i=1,2, \ldots, \mathrm{SL}, \\
\text { fit }\left(t_{N_{j}}^{\text {best }}\right) & =\max \left(\operatorname{fit}\left(t_{N_{j}}^{1}\right), \text { fit }\left(t_{N_{j}}^{2}\right), \ldots, \text { fit }\left(t_{N_{j}}^{s}\right)\right)
\end{aligned}
$$

Step 7. Produce the new solutions (new positions) $v_{N_{j}}^{\text {best }}$ for the onlookers from the solutions $t_{N_{j}}^{\text {best }}$ using the formula:

$$
v_{N_{j, i}}^{\text {best }}=t_{N_{j, i}}^{\text {best }}+\varphi_{t, i} *\left(t_{N_{t, i}}^{\text {best }}-t_{r, i}\right)
$$

And then, selected depending on $\operatorname{prob}_{i}$, and evaluate them;

Step 8. Apply the greedy selection process for the onlookers between $t_{N_{j}}^{\text {best }}$ and $v_{N_{j}}^{\text {best }}$

Step 9. Determine the abandoned solution (source food), if it exists, and replace it with a new randomly produced solution $t_{j}$ for the scout;

Step 10. Memorize the best food source position (solution) achieved so far;

Step 11. End for

Step 12. End while

\section{Membership Initialization and Local Information Aggregation}

After the optimized processing in the above Section 4, a set of thresholds is obtained through (8), and the subsequent processing will take the thresholds as the basis. Based on fuzzy theory, every pixel will be assigned with a fuzzy membership. The process is called membership initialization. On the basis of the initialization value, this paper proposes to conduct the local information aggregation based on the pixel spatial relationship so as to achieve the final segmentation results.

5.1. The Membership Initialization Using Pseudo TrapezoidShaped (PTS) Membership Function. Literature [1] summarizes the initialization methods of image fuzzy sets into two kinds: histogram based methods and centroid-based methods. Histogram based methods are the most important and the most commonly used for thresholding segmentation, but such methods need the prediction of probability distribution of histograms (like the most commonly used Gaussian distribution). Unfortunately, the probability distribution of the histograms for different kinds of images follows different probability patterns, like MR data is known to follow a Rician distribution [34], ultrasound data have been described more accurately by a mixture of Gamma distributions [1]. As a result, applicability of histogram based methods is relatively poor; in contradistinction, centroid-based methods render the shape and probability of histogram unnecessary; what only needs to be done is to select a proper centroid. As previously described, the thresholds which are obtained through (8) are set as the centroids, based on that, the fuzzy degree of each pixel is distributed through a proper function. Figure 1 provides fuzzy membership function adopted by the fuzzy entropy. In the process of initialization of image fuzzy sets, Pseudo Trapezoid-Shaped membership function (PTS) which is proposed in literature [1] will be adopted, as shown 


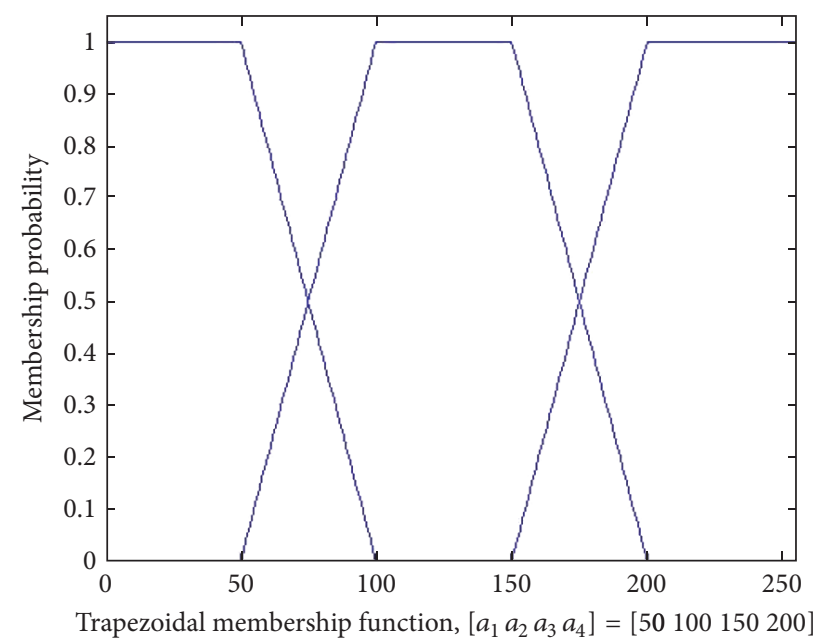

Figure 1: Fuzzy trapezoidal membership function.

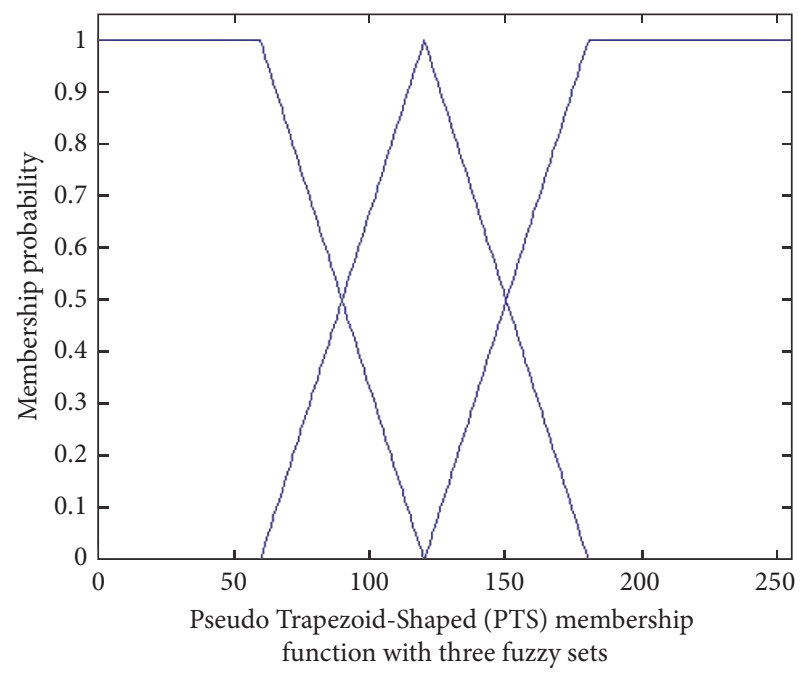

Figure 2: Fuzzy Pseudo Trapezoid-Shaped membership function.

in Figure 2. Comparing Figures 2 and 1, PTS has less fuzzy degree values of 1 which is more conducive to the use of fuzzy spatial information aggregation in the following step.

Similar to the formulation of trapezoidal membership function, in the case that there are $N$ centroids, PTS membership function can be formulated as follows:

$$
\begin{aligned}
& \mu_{0}^{\prime}(k)= \begin{cases}1, & k \leq a_{1}, \\
\frac{k-a_{2}}{a_{1}-a_{2}}, & a_{1}<k \leq a_{2}, \\
0, & k>a_{2},\end{cases} \\
& \mu_{1}^{\prime}(k)= \begin{cases}0, & k \leq a_{1}, \\
\frac{k-a_{1}}{a_{2}-a_{1}}, & a_{1}<k \leq a_{2}, \\
\frac{k-a_{3}}{a_{2}-a_{3}}, & a_{2}<k \leq a_{3}, \\
0, & k>a_{3}\end{cases}
\end{aligned}
$$

$$
\mu_{m}^{\prime}(k)= \begin{cases}0 & k \leq a_{n-1} \\ \frac{k-a_{n-1}}{a_{n}-a_{n-1}} & a_{n-1}<k \leq a_{n} \\ 1 & k>a_{n},\end{cases}
$$

where $\mu_{0}^{\prime}(k), \mu_{1}^{\prime}(k), \ldots, \mu_{m}^{\prime}(k)$ are initialization functions of pixel fuzzy membership; $a_{1}, a_{2}, \ldots, a_{n}$ are the fuzzy parameters of the Pseudo trapezoidal membership; $k$ is the gray scale value for each pixel in the test image.

Figure 2 presents the distribution methods of fuzzy degree of each pixel when there are three centroids being set. The membership of any random pixel in image $I$ in $r$ class is represented by $\mu_{r}(I(i))$; after the initialization of the image's fuzzy membership by way of PTS membership function, every pixel corresponds to a membership vector $\mu_{r}(I(i))$, as described in

$$
\mu(I(i))=\left[\begin{array}{llll}
\mu_{0}(I(i)) & \mu_{1}(I(i)) & \cdots & \mu_{m}(I(i))
\end{array}\right] .
$$

5.2. Membership Aggregation Based on Local Spatial Information. After the initialization of fuzzy membership function, one pixel is likely to be distributed into two regions at most (in (22), there are two nonzero numbers at most). Simply by adopting the biggest value, it will be enough to distribute the pixel into the corresponding region, but such methods only consider the single pixel's fuzzy degree, to the neglect of the pixel spatial relationship. Therefore, analyzing the local information aggregation of adjacent pixels will improve segmentation precision so that the isolated pixels can be removed.

Image segmentation is in essence the aggregation of adjacent pixels of the same features. The biggest drawback of thresholding segmentation lies in the fact that it only takes the gray scale features of the test image to the neglect of spatial relationship. In the process of initialization of fuzzy membership function, each pixel will be assigned with one or two fuzzy values. These indeterminate values provide optimal prerequisites for local aggregation. By means of average and median aggregation of the fuzzy degrees of the pixels in neighborhood, the quality of image segmentation can be improved effectively.

When there exists $N$ surrounding regions for each pixel, the value of the fuzzy degree of each pixel can maximize the fuzzy relevance of pixels in surrounding regions by average and median filtering. As depicted in literature [1], there exist three methods for local fuzzy aggregation.

(a) Median Aggregation. The fuzzy membership degree of each pixel will get through median filter in $N$ surrounding regions, which can be formulated as follows:

$$
\begin{aligned}
& \mu^{\text {aggr }}(I(i)) \\
& \quad=\operatorname{median}_{I(i) \in N(I(i))}\left\{\mu_{0}(I(i)) \quad \mu_{1}(I(i)) \cdots \mu_{m}(I(i))\right\},
\end{aligned}
$$


where $\mu^{\text {aggr }}()$ represents fuzzy membership degree after aggregation, median \{\} represents the median operations of vector quantity, and $N(I(i))$ is the number of $N$ neighboring pixels for number $i$ pixel.

(b) Average Aggregation. In the scope of the neighborhood for each pixel (like $3 * 3$ ), search for the solution which includes the average values of all pixels (including itself) and take them as the fuzzy membership of the present pixel, as described in

$$
\mu^{\text {aggr }}(I(i))=\frac{1}{N(I(i))} * \sum_{I(i) \in N(I(i))} \mu(I(i)) .
$$

(c) Iterative Averaging Aggregation. Compared to median aggregation, average aggregation can make better use of neighboring pixel information. Therefore the latter performs better in removing isolated pixels. But the aggregation capability with one-time iteration is limited; therefore, iterative averaging aggregation can strengthen aggregation effects. At the same time we should bear in mind that too large the number of iterations will make the segmentation boundary blurry; consequently, it is necessary to set different weights for adjacent pixels with different spatial distances as depicted in (25). Accordingly, the iterative formulation is shown in (26), where $\mu^{\text {aggr }}\left(I(i)_{t}\right)$ represents the computational results of (24) when iterations occur once.

$$
\begin{aligned}
w & =\frac{1}{3} *\left[\begin{array}{ccc}
0 & 0.5 & 0 \\
0.5 & 1 & 0.5 \\
0 & 0.5 & 0
\end{array}\right], \\
\mu^{\mathrm{aggr}}\left(I(i)_{t+1}\right) & =w * \mu^{\mathrm{aggr}}\left(I(i)_{t}\right) .
\end{aligned}
$$

After the fuzzy optimization and fuzzy aggregation, each pixel in the test image will get a vector by (22). In the set of vector quantities, selecting the biggest fuzzy value will determine which region the pixel belongs to. The whole procedures of computations are represented by Algorithm 2.

Algorithm 2 (fuzzy multilevel image thresholding).

Step 1. Initialization of the number of region $N$, and convert the input image to gray scale and calculate the histogram of grayscale image.

Step 2. Set the number of fuzzy parameters $2 * N$ which is used in fuzzy trapezoidal membership function (Figure 1). Provide the number of thresholds $m$ and maximum cycle number CN.

Step 3. Use QABC (as described in Algorithm 1) to compute the fuzzy parameters. The objective function follows (7) which merges the fuzzy theory and Kapur's entropy, as described in Section 3.2.

Step 4. The soft threshold calculated by fuzzy parameters obtained in Step 3.

$$
\begin{gathered}
t_{0}=\frac{a_{1}+a_{2}}{2}, \\
t_{1}=\frac{a_{3}+a_{4}}{2} \\
\vdots \\
t_{m}=\frac{a_{n-1}+a_{n}}{2} .
\end{gathered}
$$

Step 5. Regard the soft thresholds as the centroids, assigning membership to each pixel using fuzzy Pseudo TrapezoidShaped membership function (Figure 2).

Step 6. According to the local neighborhood information, perform aggregation operations on the membership degree of each pixel as described in Section 5.2

Step 7. According to the results of aggregation, determining the region for each pixel by way of maximum value.

Step 8. According to the regional labels, output the segmentation result.

\section{Experiments and Result Discussions}

This paper, on the basis of numerical comparative experiments, through contrasts of segmented images, data, and graph analysis, testifies to the superiority of the proposed algorithm. From relevant literature, it can be seen that some methods are superior to others. The present paper will compare the proposed algorithm with the state-of-theart methods; proven inferior ones will be sidelined. In the following sections, the proposed algorithm will be compared to Electromagnetism method, standard $\mathrm{ABC}$, and the latest intelligent optimization that takes Kapur's entropy as the objective function proposed in literature [7, 27, 35]. Electromagnetism optimization with Kapur's entropy as the objective function is so far the newest intelligent optimization. PDE is one of the most effective fuzzy entropy thresholding segmentation methods. The comparison with literature [35] is mainly to testify that MQABC outperforms standard ABC.

6.1. The Discussion of Initialization Parameters and Convergence in MQABC. The proposed algorithm has been tested under a set of benchmark images. Some of these images are widely used in the multilevel image segmentation literature to test different methods (Lena, Baboon, and so forth) [7, 8, 35]. Others are chosen purposefully from the Berkeley Segmentation Data Set and Benchmarks 500 (BSDS500 for short, see [36]), as shown in Figure 3. The experiments were carried out on a LENOVO Laptop with Intel(R) Core i3 processor and $4 \mathrm{~GB}$ memory. And the algorithm was developed via the signal processing toolbox, image processing toolbox, and global optimization toolbox of MatlabR2011b. the parameters used for ABC algorithms [35] were presented in Table 1. In order to test the specific effects of these parameters in MQABC, many experiments have been conducted. Figure 4 presents the convergence curve of objective functions with 
TABLE 1: Parameters used for MQABC.

\begin{tabular}{lccccc}
\hline Parameters & Swam size & Number of iterations & Lower bound & Upper bound & Max trial limit \\
\hline Value & 30 & 200 & 1 & 256 & 10 \\
\hline
\end{tabular}

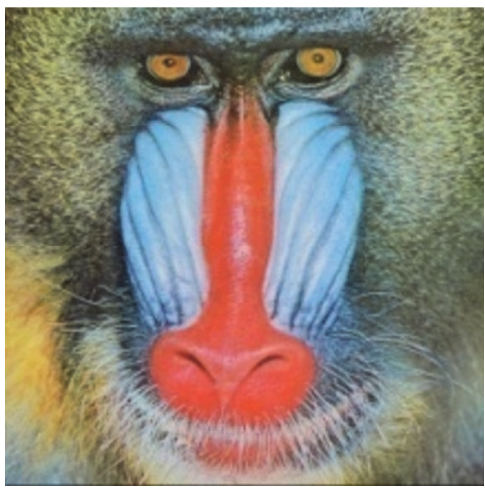

(a)

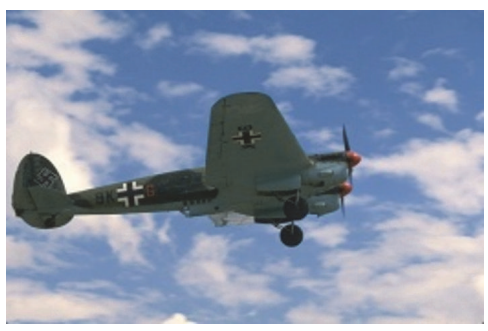

(c)

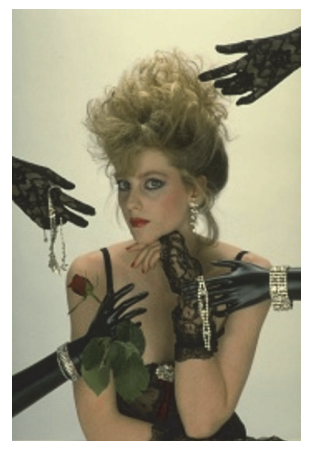

(e)

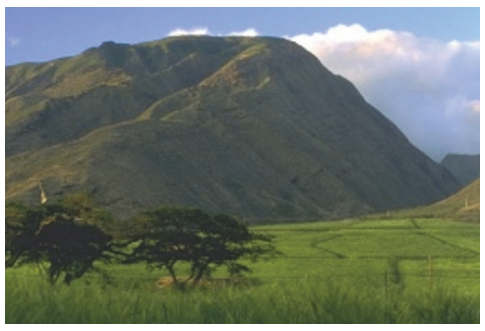

(g)

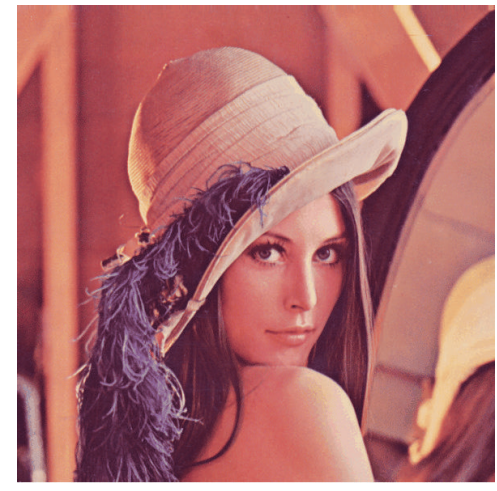

(b)

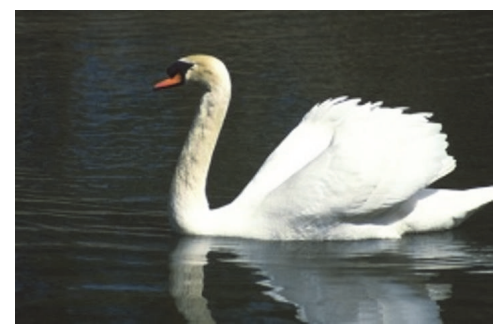

(d)

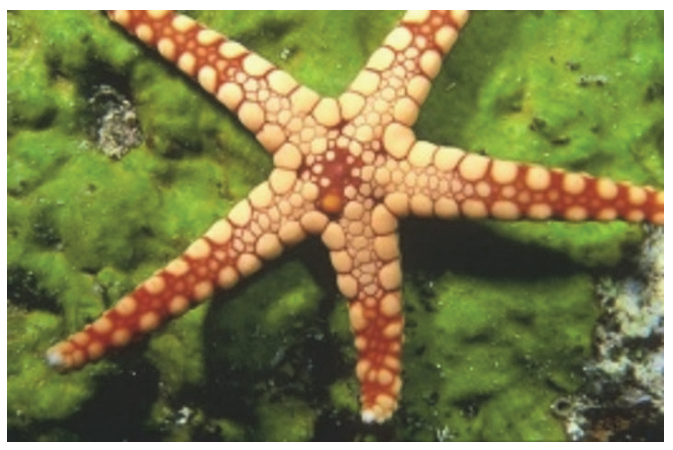

(f)

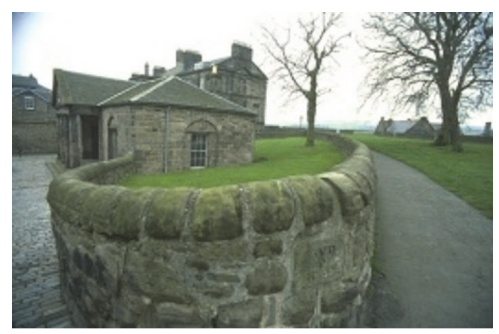

(h)

Figure 3: The original images and their histograms: (a) Baboon, (b) Lena, (c) Aeroplane, (d) Swan, (e) Ladyhand, (f) Starfish, (g) Mountain, and (h) Building. 
TABLE 2: Comparison of image segmentation quality with different swarm size.

\begin{tabular}{lccccc}
\hline Swam size & 10 & 20 & 30 & 40 & 50 \\
\hline PSNR & 20.3650 & 20.4675 & 20.7193 & 20.4243 & 20.4071 \\
\hline
\end{tabular}

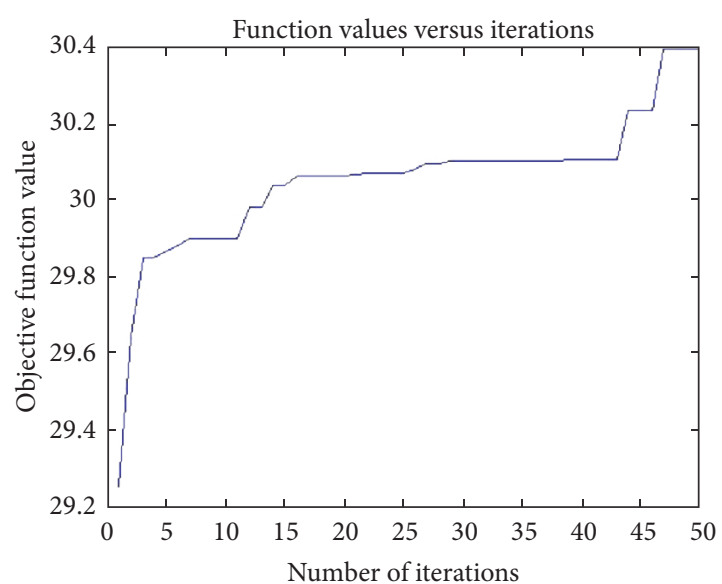

50

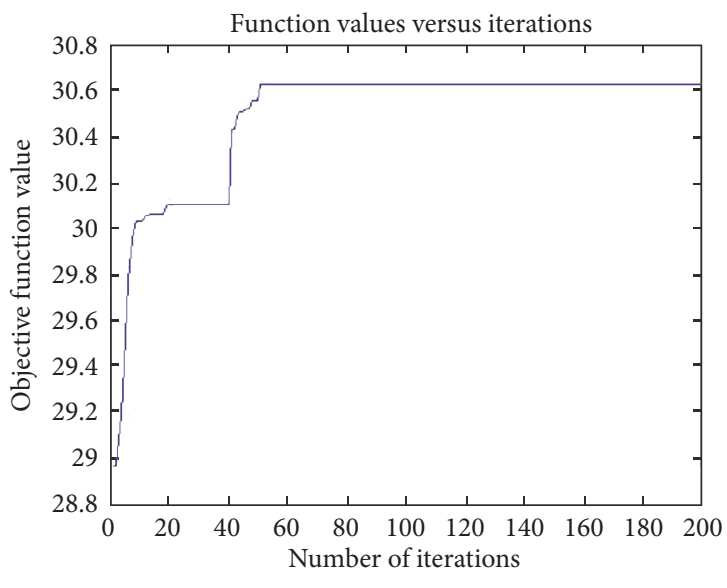

200

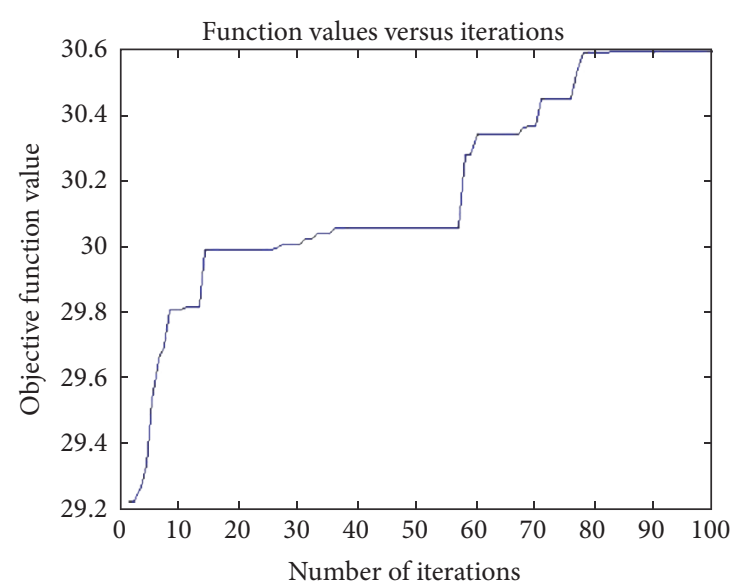

100

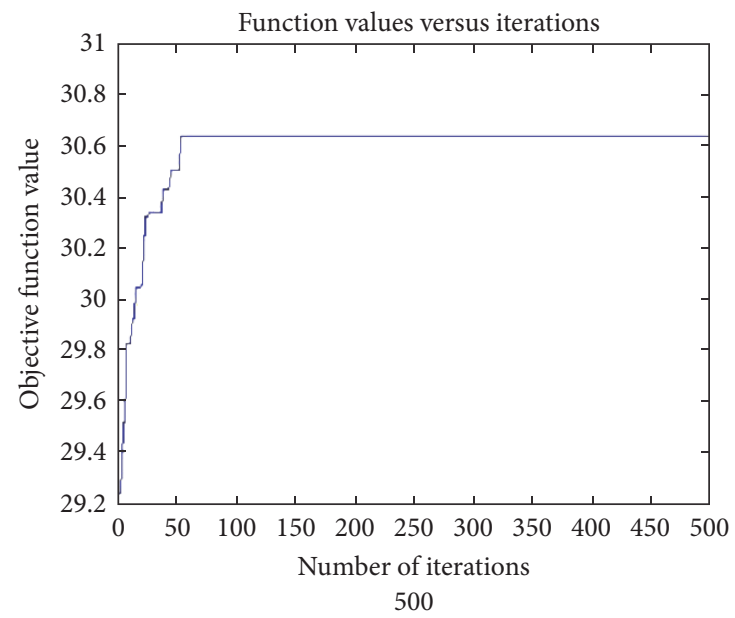

FIGURE 4: The comparison of convergence with different number of iterations.

iterations ranging from 50 to 500 . When the iterations range from 50 to 150 , the convergence is not so apparent. But when the iterations reach 200 , the convergence is evident enough. However, when the number of iterations increases further, the convergence speed is hardly improved. Table 2 presents the assessment parameters for the segmentation quality of image Baboon with different swarm-sizes (detailed PSNR is provided at Section 6.4). From the table, it can be seen that when swarm-size reaches 30 , the quality of image segmentation reaches the highest. At the same time, we also test the parameter changes of max trial limit. In the following tests, the biggest parameter value is 7 , but most of them are below 5. Therefore, the parameters presented in Table 1 can be applied directly to MQABC.

In order to highlight the convergence performance of MQABC visually, Figure 5 shows the convergence curves of MQABC and ABC for test images Baboon, Lena, and starfish when the thresholds are 5, respectively. From the curve lines, MQABC not only reaches the optimal objective earlier, but also is speedier toward the optima. More importantly, compared with $\mathrm{ABC}$ algorithm, the objective value obtained by MQABC is equal or even bigger, which means MQABC can get better thresholds. Therefore, MQABC shows better convergence performance and processing results.

6.2. The Fuzzy Image Segmentation Result Based on MQABC with Different Thresholds. In order to make comparisons with other thresholding segmentation algorithms, one of the most popular performance indicators PSNR (the peak-tosignal ratio) is employed to compare the segmentation results between the original image and the segmented image. It is defined as in

$$
\operatorname{PSNR}(i, j)=20 \log _{10}\left(\frac{255}{\operatorname{RMSE}(i, j)}\right),
$$

where RMSE is the root mean-squared error.

In order to compare the influence of different thresholds and different aggregation methods, we conducted numerical 


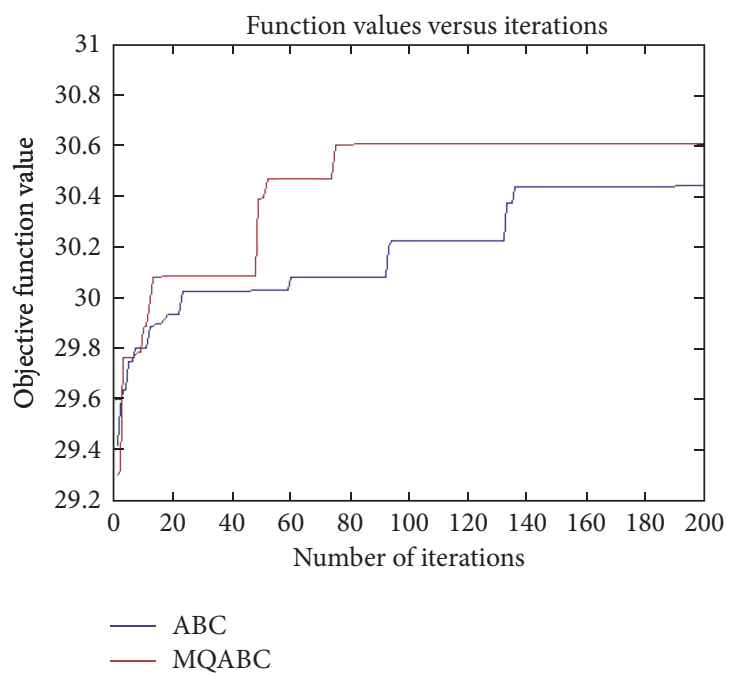

(a) Baboon with 5 th

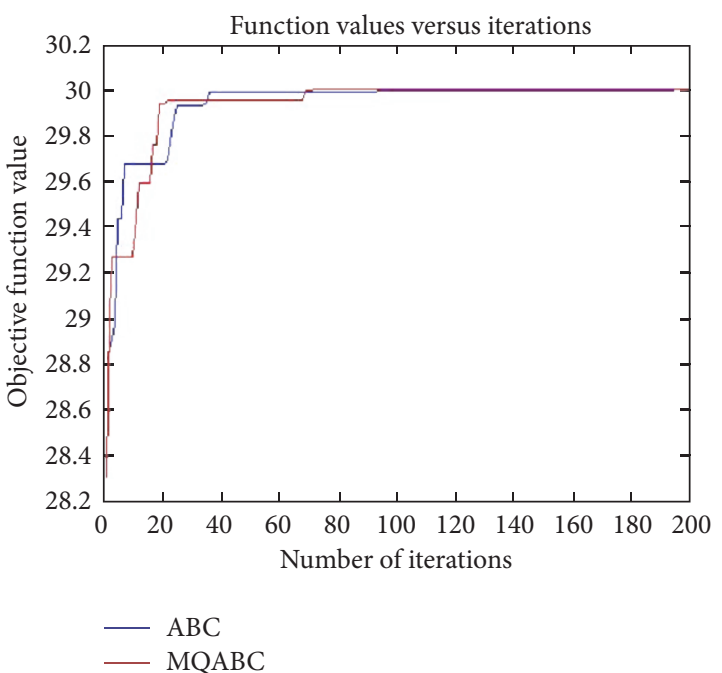

(b) Lena with 5 th

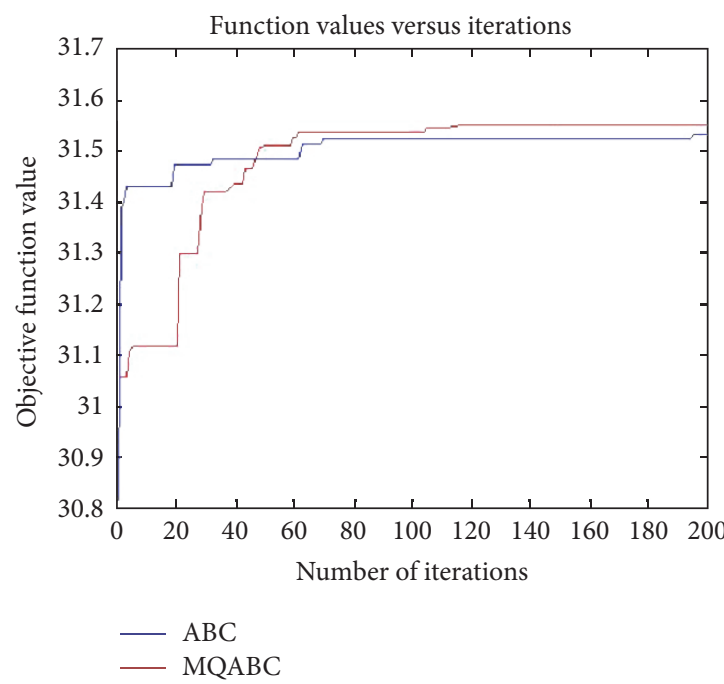

(c) Starfish with 5 th

FIGURE 5: The comparison of convergence (a)-(c) with 5 thresholds, respectively.

experimental tests. Visual effects and qualitative data indicate that with the increase of the number of thresholds, image segmentation results will get finer. Note that the number of thresholds cannot increase without limit. The number of information units in each image is finite; too much thresholds cannot improve segmentation results; on the contrary it will add to the complexity. Based on the data sets adopted by this paper, we test the segmentation results when the number of thresholds ranges from 2 to 5 and adopt three different membership aggregations to conduct neighboring aggregations. The experimental results indicate that in the case of the same number of thresholds, the median aggregation gets the best segmentation results. When the average aggregation is compared to iterative average aggregation, in most cases, iterative average aggregation performs better except for some extremely rare occasions. Table 3 presents different PSNR value of Baboon image with different thresholds and aggregation. Figure 6 shows the segmentation results for the images in Table 3. From Figure 6, we can see that with the increase of the number of thresholds, the segmentation results get finer gradually, but the visual effects are not obvious enough for different aggregation methods. To compensate for the insufficiency of visual comparisons, Table 3 clearly indicates that the median aggregation is far superior to the other aggregation methods.

Figures 7 and 8 show the segmentation results of the original images in Figure 3 after median aggregation is done with the thresholds being 2, 3, 4, and 5, respectively. Visually, it is adequate enough to discriminate the object from its background when the thresholds are 2 . With the increase of the number of thresholds, the segmentation results get finer. In actual applications, we can vary the number of thresholds to obtain the desired output in response to different functions and scenes. Surely, if the size of the image is much larger or needs dividing into more regions, to that end, it is quite necessary to increase the number of thresholds. What needs 
TABLE 3: Comparison of segmentation quality with different thresholds and aggregation.

\begin{tabular}{lcccc}
\hline$M$ & 2 & 3 & 4 & 5 \\
\hline Median aggregation & 18.8793 & 19.9228 & 20.4195 & 21.0405 \\
Average aggregation & 18.2370 & 18.7917 & 19.3352 & 19.7773 \\
Iterative averaging & 18.6391 & 19.5356 & 20.0112 & 20.5206 \\
\hline
\end{tabular}

$M$
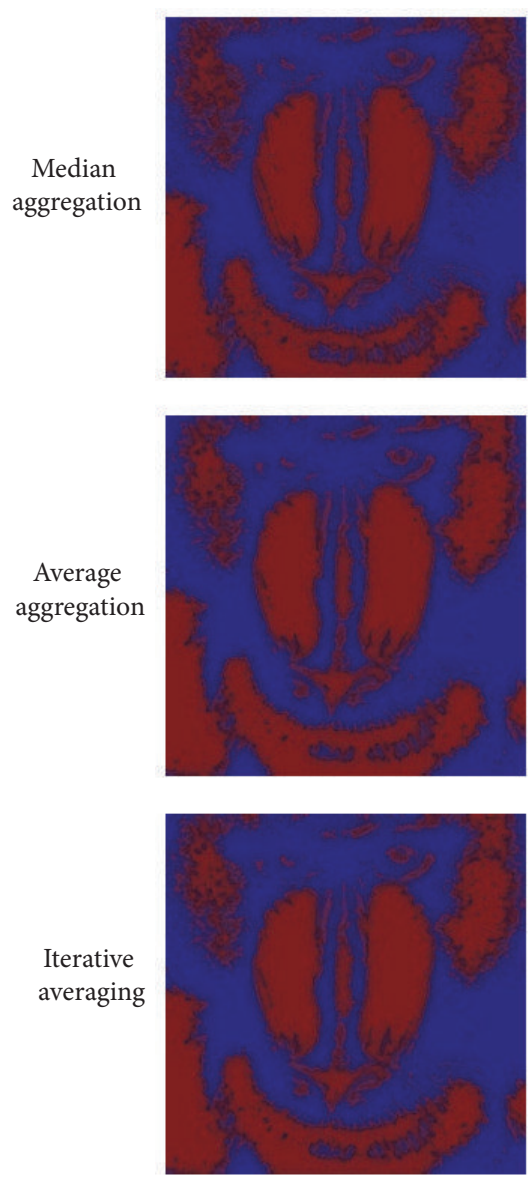

3
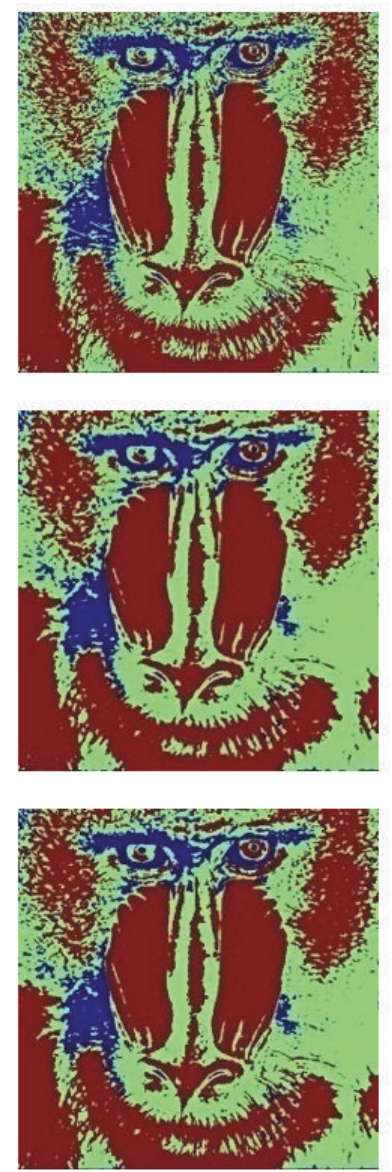

4
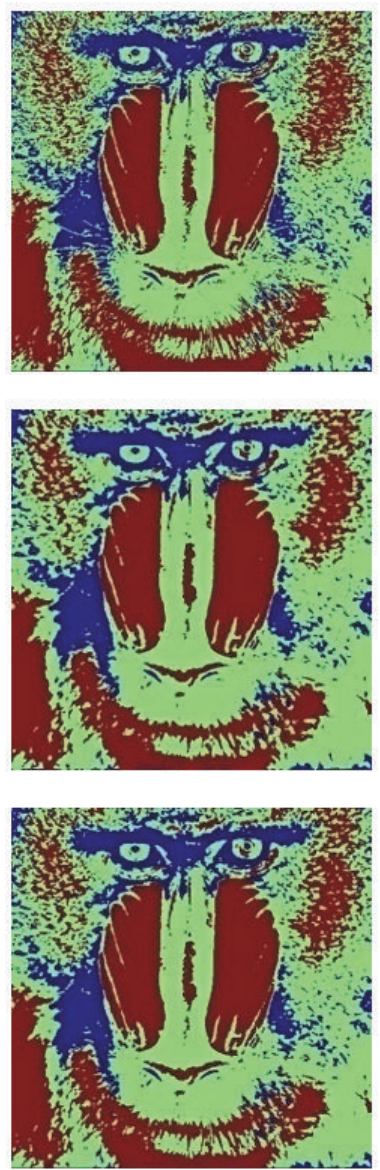

5
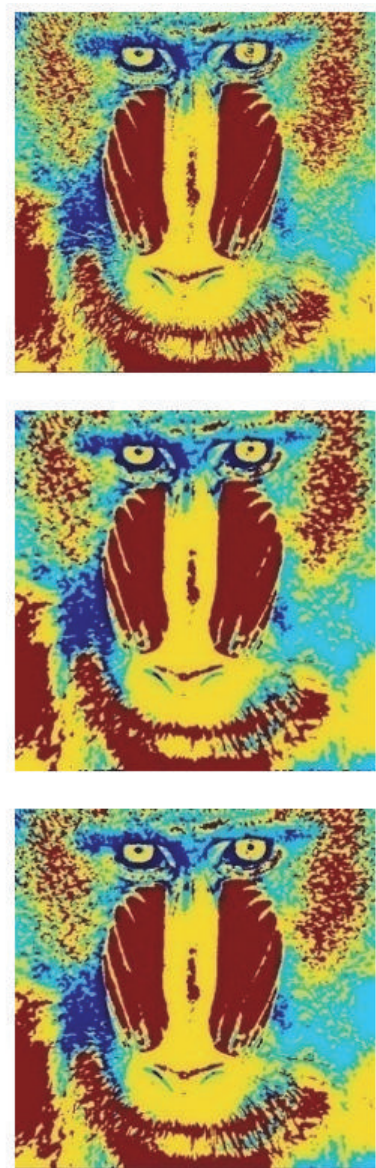

FIGURE 6: The results of image segmentation with different thresholds and aggregation.

to be done in the proposed method is just to set the number of thresholds $M$ to get the desired results. Compared with other segmentation methods, it is hard to tell visually the difference of segmentation results. As for more detailed and comprehensive assessment concerning the segmentation results and performance, we will provide qualitative analysis in the subsequent sections.

6.3. Comparison of Best Objective Function Values and Their Corresponding Thresholds. In this section, the results of best objective function values and their corresponding thresholds acquired by various images are discussed. Table 4 depicts the number of thresholds, objective values, and the corresponding optimal thresholds obtained by EMO, ABC, and MQABC methods. From the tables, it can be observed that all the images when processed by different methods have obtained relatively high objective function values. Notably, with the increase of the number of thresholds, the three methods all get high objective function values. Generally, MQABC and EMO obtain similar and even higher objective function values, while $\mathrm{ABC}$ appears a little inferior, but the difference range is less than 0.1 .

Table 4 provides only the comparisons of the best objective function values and their corresponding thresholds between EMO, ABC, and MQABC. After the introduction of fuzzy entropy, we also tested the optimized results in terms of thresholds and objective function values based on DE (differential evolution) and FMQABC.

Tables 3 and 4 indicate that MQABC and FMQABC are able to obtain relatively good objective function values. Although, in some cases, these objective function values appear a little smaller in comparison with EMQ and FDE, the difference is insignificantly trivial. This paper lays more 
TABLE 4: Comparison of best objective function values and their corresponding thresholds between EMO, ABC, and MQABC.

\begin{tabular}{|c|c|c|c|c|c|c|c|}
\hline \multirow{2}{*}{ Image } & \multirow{2}{*}{$M$} & \multicolumn{3}{|c|}{ Optimum thresholds } & \multicolumn{3}{|c|}{ Best objective function values } \\
\hline & & EMO & $\mathrm{ABC}$ & MQABC & EMO & $\mathrm{ABC}$ & MQABC \\
\hline \multirow{4}{*}{ Baboon } & 2 & 78143 & 80144 & 80144 & 17.6799 & 17.6802 & 17.6802 \\
\hline & 3 & 46100153 & 46101153 & 46100153 & 22.1331 & 22.1331 & 22.1331 \\
\hline & 4 & 46100153235 & 45100153236 & 45100152235 & 26.5254 & 26.5249 & 26.5265 \\
\hline & 5 & 3475115160235 & 3475116161236 & 3374115162235 & 30.6432 & 30.4394 & 30.6509 \\
\hline \multirow{4}{*}{ Lena } & 2 & 97164 & 97164 & 97164 & 17.8234 & 17.8234 & 17.8234 \\
\hline & 3 & 81126177 & 83127178 & 82126176 & 22.1102 & 22.1091 & 22.1201 \\
\hline & 4 & 1682127177 & 1687123174 & 1683125176 & 26.1107 & 26.1064 & 26. 1101 \\
\hline & 5 & 166497137179 & 166596133178 & 166497137178 & 30.0052 & 29.9870 & 30.0029 \\
\hline \multirow{4}{*}{ Aeroplane } & 2 & 54161 & 54161 & 54161 & 17.8618 & 17.8618 & 17.8618 \\
\hline & 3 & 54157229 & 54134186 & 54132184 & 22.3106 & 22.2964 & 22.3112 \\
\hline & 4 & 54127167206 & 47109165227 & 48110165228 & 26.3540 & 26.6389 & 26.6427 \\
\hline & 5 & 4577109155202 & 4196109168229 & 45109149189229 & 30.8109 & 30.8083 & 30.9162 \\
\hline \multirow{4}{*}{ Swan } & 2 & 66150 & 66150 & 66150 & 18.1274 & 18.1274 & 18.1274 \\
\hline & 3 & 64132200 & 64133198 & 64131199 & 22.9607 & 22.9621 & 22.9887 \\
\hline & 4 & 64117161210 & 64117164212 & 64117162210 & 27.3410 & 27.3493 & 27.3509 \\
\hline & 5 & 63108140179218 & 6397132170212 & 6398134174212 & 31.2520 & 31.2512 & 31.2654 \\
\hline \multirow{4}{*}{ Ladyhand } & 2 & 86171 & 86171 & 86171 & 18.4477 & 18.4477 & 18.4477 \\
\hline & 3 & 63116182 & 64118180 & 61115180 & 22.9799 & 22.9883 & 22.9890 \\
\hline & 4 & 5295137180 & 5396136180 & 5196138182 & 27.1901 & 27.1956 & 27.1958 \\
\hline & 5 & 5297136168202 & 5698137168202 & 5598137174204 & 31.1297 & 31.1302 & 31.1321 \\
\hline \multirow{4}{*}{ Starfish } & 2 & 90170 & 90170 & 90170 & 18.7518 & 18.7518 & 18.7518 \\
\hline & 3 & 73131185 & 73127182 & 72127185 & 23.3105 & 23.3192 & 23.3241 \\
\hline & 4 & 68117166208 & 63112159207 & 67118167206 & 27.5621 & 27.5509 & 27.5675 \\
\hline & 5 & 5796135173211 & 5594132170210 & 5390130170210 & 31.5536 & 31.5338 & 31.5519 \\
\hline \multirow{4}{*}{ Mountain } & 2 & 117196 & 117196 & 117196 & 17.6737 & 17.6737 & 17.6737 \\
\hline & 3 & 62119196 & 62119196 & 62119196 & 22.1255 & 22.1255 & 22.1255 \\
\hline & 4 & 2062119196 & 2062120198 & 2062119194 & 26.4474 & 26.4338 & 26.4427 \\
\hline & 5 & 206196137197 & 206096139203 & 2061100159204 & 30.5513 & 30.5448 & 30.5562 \\
\hline \multirow{4}{*}{ Building } & 2 & 90156 & 90156 & 90156 & 17.8071 & 17.8071 & 17.8071 \\
\hline & 3 & 84139191 & 84139190 & 83141194 & 22.3895 & 22.3893 & 22.3876 \\
\hline & 4 & 2084141193 & 2084141192 & 2083139192 & 26.7108 & 26.7105 & 26.7106 \\
\hline & 5 & 2081131170213 & 2083136177218 & 2081134173214 & 30.7119 & 30.8631 & 30.8983 \\
\hline
\end{tabular}

emphasis on time efficiency of MQABC and FMQABC; detailed comparisons are presented in Section 6.5.

6.4. Comparison of Fuzzy Multilevel Image Segmentation Result and Quality Assessment by PSNR. Figures 7 and 8 demonstrate the segmentation results of FMQABCA. In order to further compare the proposed method with MQABC and other fuzzy optimized methods, Figure 9 presents the segmentation results of FDE, FMQABC, and FMQABCA with different thresholds. For the sake of convenience, the results in Figure 7 will have to be grayscaled; the gray level in the same region will be replaced by the average value. Analyzed visually, all the three methods get relatively fine segmentation results, but it is hard to tell visually which one is superior or inferior.

In order to further compare the advantages and disadvantages between EMO, ABC, MQABC, FDE, FMQABC, and FQMABCA, Table 5 demonstrates PSNR values these methods can achieve on fuzzy entropy, which indicate that MQABC gets the high assessment value, testifying to the superiority of MQABC in segmentation quality results. Furthermore, when the number of thresholds increases, MQABC gets better PSNR values. The comparison of fuzzy entropy (FDE, FMQABC, and FQMBCA) with nonfuzzy entropy (EMO, ABC, and MQABC) shows that in most of the cases, PSNR value is higher when fuzzy entropy is applied; noticeably FMABCA is far superior to the rest of the methods. The FMQABCA method gets the highest increase of $42 \%$ for the Swan image when $M=3$; the lowest also increases by $0.8 \%$ for the Aeroplane image when $M=4$. On average, the FMQABCA method shows more than $12 \%$ increase on the PSNR value for the eight figures.

6.5. Comparison of the Characteristics of Running Time. On the prior condition that segmentation quality is guaranteed, we examine the running time to test the performance. 

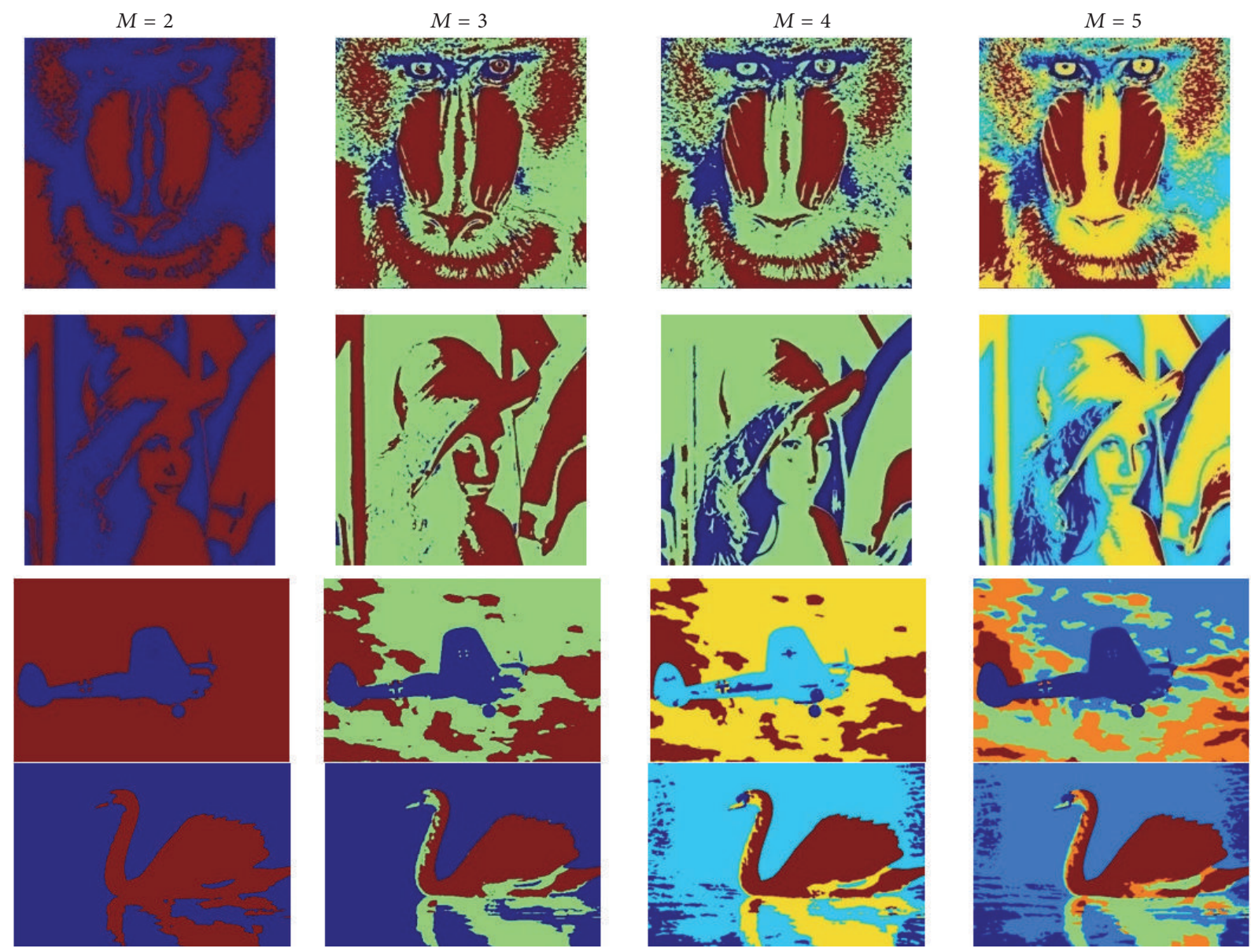

FIGURE 7: The segmentation results of (a)-(d) in Figure 1.
This is the biggest highlight of this paper. As shown in Table 1, the experiments are set to the same number of iterations for testing. But in order to obtain the operating time for convergence as described in Table 6, when the fitness function remains unchanged for 20 consecutive times, the experimental iteration is forced to be terminated. Moreover, in order to better ensure the effectiveness of MQABC, its consecutive times are set to 50 specifically. Table 6 presents data concerning running time to convergence. The running time that EMO takes is much longer than the others. First, when comparing $\mathrm{ABC}$ with MQABC, we find that MQABC method gets much less running time than the $A B C$. And the highest increase of $12 \%$ is for the Lena image with $M=5$; the average also increases by $7.7 \%$ with $M=5$ and $4.8 \%$ with $m=4$. Then, the comparison of fuzzy methods to nonfuzzy methods shows that the running time for fuzzy methods takes a little longer, but if we take the segmentation quality assessment (PSNR) into account as shown in Table 5 the segmentation result is much better. All taken together, fuzzy methods are much more valuable.

In the three fuzzy methods, when FDE is compared with FMQABC and FBCA, it shows the latter two enjoy higher time efficiency significantly. The FMQABC method gets the highest increase of $53 \%$ for the Swan image when $M=4$; the lowest also increases by $28 \%$ for the Lena image when $M=5$. On average, the FMQABC method shows more than $44 \%$ of the time improvement for the eight figures when $M=4$ and more than $41 \%$ when $M=5$. From the perspective of FMQABCA, the maximum and minimum running time increase by $40 \%$ and $14 \%$ for the Aeroplane and Lena image, respectively, when $M=4$. And when $M=5$, the maximum and minimum running time increase by $38 \%$ and $21 \%$ for the Lena and Aeroplane image, respectively. On average, the FMQABCA method shows more than $28 \%$ of the time improvement for the eight figures when $M=4$ and more than $26 \%$ when $M=5$. Therefore, the FMQABC and FMQABCA methods ensure the optimal threshold so as to obtain the best time efficiency.

From Tables 4-6 and Figures 4-9, together with visual effect analysis, it can be seen that FMQABCA delivers better segmentation results and enjoys obvious advantages in running time. More importantly, MQABC is far superior to $\mathrm{ABC}$ over convergence performance. Therefore it can be safely concluded that FMQABCA is a desirable multilevel segmentation method with high speed and of high quality. 

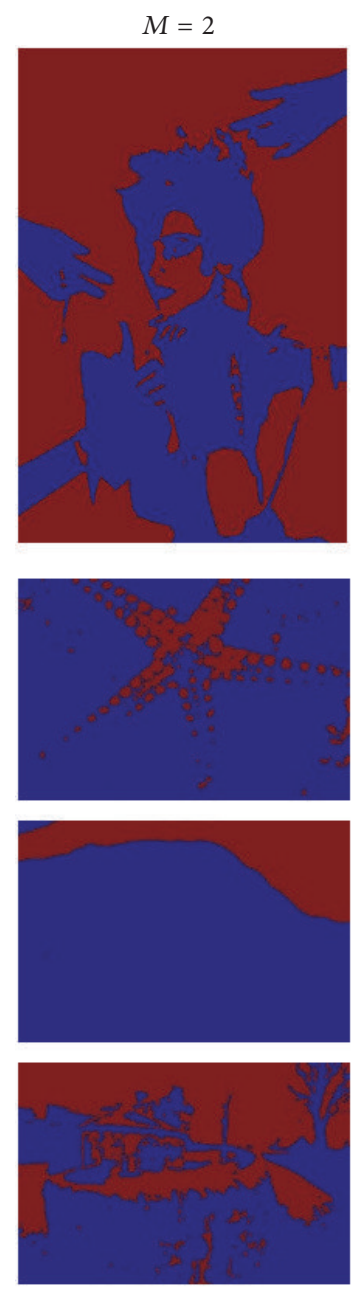

$M=3$
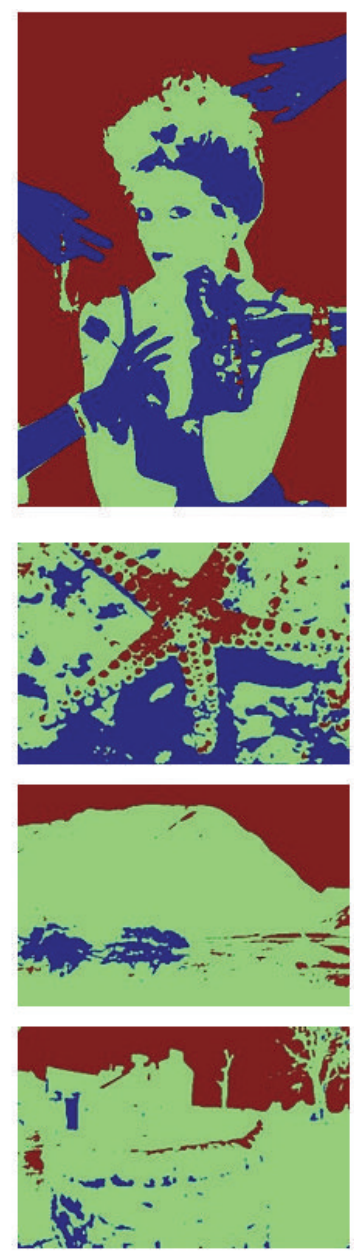

$M=4$
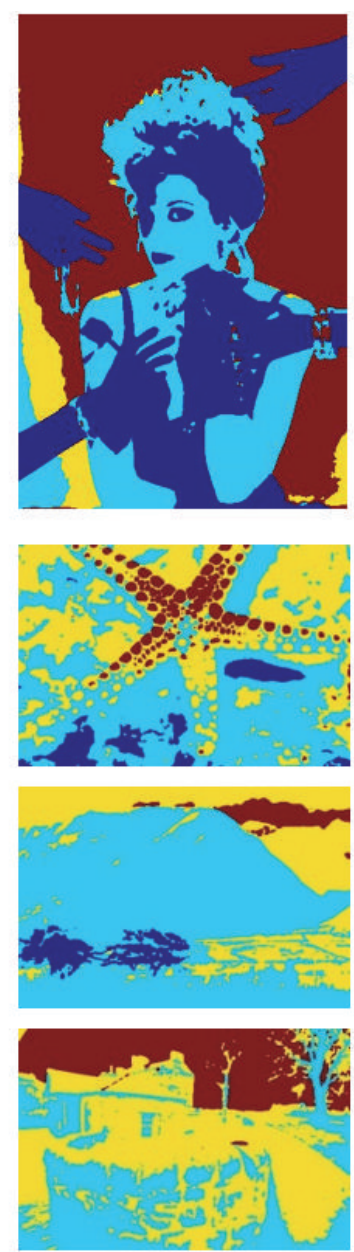

$M=5$
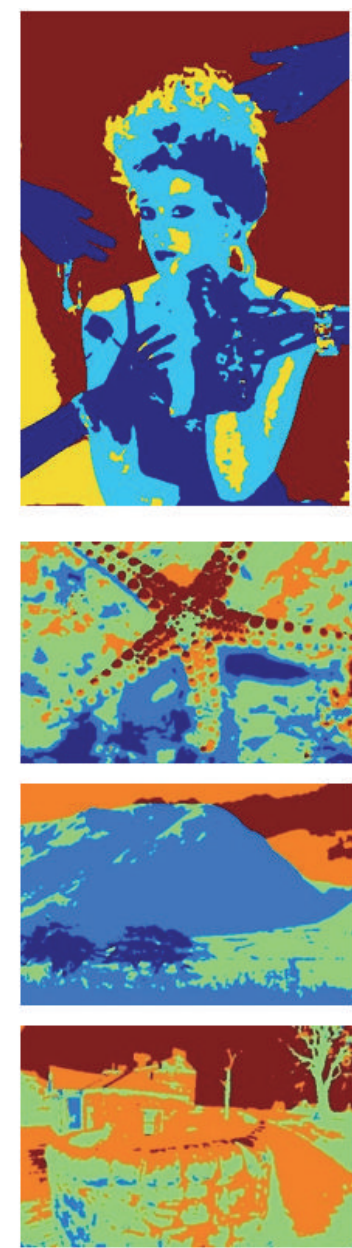

Figure 8: The segmentation results of (e)-(h) in Figure 1.

\section{Conclusion}

In this paper, MQABC has been employed to optimize fuzzy Kapur's entropy in order to obtain a set of thresholds. Based on the set values, a set of fuzzy degrees is assigned to each pixel through Pseudo Trapezoid-Shaped membership function, and then aggregation operation is conducted in the way that fuzzy degrees of neighborhood are aggregated to achieve the final segmentation. When compared with $A B C$, MQABC, by improving on the distance strategies in the onlooker bee phase, not only achieves the higher objective function and betters PSNR value, but also shows obvious improvement in convergence performance and running time. In the process of fuzzy aggregation, FMQABCA gets higher PSNR values obtained by median, average, and iterative methods than EMO, ABC, MQABC, FDE, and FMQABC and show more efficiency in running time than EMO and FDE. Although compared with $\mathrm{ABC}, \mathrm{MQABC}$, and FMQABC, FMQABCA takes a slightly longer time, but such a little price is completely acceptable if we take the high quality of image segmentation into account. In conclusion, FMQABC and FMQABCA are able to obtain better image segmentation quality and also show obvious superiority in terms of running time and convergence speed.

\section{Competing Interests}

The authors declare that there are no competing interests regarding the publication of this paper.

\section{Acknowledgments}

This work was supported in part by the National Natural Science Foundation of China (nos. 61300239, 71301081, and 61572261), The Postdoctoral Science Foundation (nos. 2014M551635 and 1302085B), the Innovation Project of graduate students Foundation of Jiangsu Province (KYLX15_0841), Higher Education Revitalization Plan Foundation of Anhui Province (nos. 2013SQRL102ZD, KJ2016A554, KJ2016A556, 2015xdjy196, and 2015jyxm728), Natural Science Fund for Colleges and Universities in Jiangsu Province (no. 16KJB520034), and a Project Funded by the Priority Academic Program Development of Jiangsu Higher 
TABLE 5: PSNR metrics of the test images segmented with different threshold.

\begin{tabular}{|c|c|c|c|c|c|c|c|}
\hline \multirow{2}{*}{ Image } & \multirow{2}{*}{$M$} & \multicolumn{6}{|c|}{ PSNR } \\
\hline & & EMO & $\mathrm{ABC}$ & MQABC & FDE & FMQABC & FMQABCA \\
\hline \multirow{4}{*}{ Baboon } & 2 & 15.9947 & 16.0070 & 16.0070 & 15.9533 & 16.2043 & 18.8793 \\
\hline & 3 & 18.5921 & 18.5921 & 18.5921 & 17.8855 & 19.1648 & 19.9228 \\
\hline & 4 & 18.5921 & 18.5435 & 18.6718 & 17.0614 & 19.8360 & 20.4195 \\
\hline & 5 & 20.5234 & 20.5058 & 20.7193 & 19.5717 & 21.0266 & 21.0405 \\
\hline \multirow{4}{*}{ Lena } & 2 & 14.5901 & 14.5901 & 14.5901 & 16.7293 & 16.6456 & 18.6328 \\
\hline & 3 & 17.2122 & 17.1178 & 17.2328 & 17.8298 & 17.8884 & 19.0366 \\
\hline & 4 & 18.5488 & 18.3798 & 18.6011 & 18.3482 & 19.3349 & 20.9990 \\
\hline & 5 & 20.3250 & 20.2440 & 20.3360 & 20.8519 & 20.9714 & 22.2737 \\
\hline \multirow{4}{*}{ Aeroplane } & 2 & 12.7187 & 12.7187 & 12.7187 & 14.5518 & 15.3454 & 16.9651 \\
\hline & 3 & 15.9198 & 16.1541 & 16.2681 & 18.4402 & 18.3086 & 20.0782 \\
\hline & 4 & 18.4136 & 18.6846 & 18.7095 & 21.0749 & 20.6326 & 21.2494 \\
\hline & 5 & 19.1549 & 18.6876 & 21.0436 & 21.2442 & 21.1950 & 21.5475 \\
\hline \multirow{4}{*}{ Swan } & 2 & 14.8785 & 14.8785 & 14.8785 & 16.2123 & 16.3309 & 17.3657 \\
\hline & 3 & 16.2369 & 16.2373 & 16.2407 & 16.2334 & 17.3286 & 23.0785 \\
\hline & 4 & 16.6445 & 16.6571 & 16.6953 & 16.7511 & 16.9194 & 23.2771 \\
\hline & 5 & 16.9943 & 16.9482 & 17.0144 & 23.3818 & 23.7829 & 24.6911 \\
\hline \multirow{4}{*}{ Ladyhand } & 2 & 15.4928 & 15.4928 & 15.4928 & 15.7940 & 15.8844 & 16.9998 \\
\hline & 3 & 17.5422 & 17.5446 & 17.5744 & 17.9525 & 17.9778 & 20.4394 \\
\hline & 4 & 18.7518 & 18.7950 & 18.9135 & 19.0744 & 20.5095 & 21.3241 \\
\hline & 5 & 20.6768 & 20.7171 & 20.7392 & 20.7710 & 21.7443 & 22.0850 \\
\hline \multirow{4}{*}{ Starfish } & 2 & 14.3952 & 14.3952 & 14.3952 & 17.0598 & 16.5631 & 17.3641 \\
\hline & 3 & 16.9727 & 17.1199 & 17.1430 & 17.3605 & 17.9680 & 19.4480 \\
\hline & 4 & 18.1811 & 18.4836 & 18.5054 & 18.5546 & 18.5492 & 19.7407 \\
\hline & 5 & 20.0649 & 20.2304 & 20.3400 & 21.2142 & 21.4146 & 21.8224 \\
\hline \multirow{4}{*}{ Mountain } & 2 & 10.8782 & 10.8782 & 10.8782 & 13.2357 & 15.0018 & 17.6530 \\
\hline & 3 & 17.4652 & 17.4652 & 17.4652 & 21.6381 & 21.6748 & 21.8547 \\
\hline & 4 & 18.0535 & 18.0726 & 18.2056 & 20.1892 & 20.9634 & 22.4085 \\
\hline & 5 & 20.6893 & 20.4889 & 21.7589 & 20.3026 & 20.4539 & 23.3328 \\
\hline \multirow{4}{*}{ Building } & 2 & 14.7242 & 14.7242 & 14.7242 & 12.2905 & 13.9163 & 15.3027 \\
\hline & 3 & 16.2205 & 16.2261 & 16.2340 & 18.1586 & 17.8589 & 18.6818 \\
\hline & 4 & 17.9914 & 18.2581 & 18.3348 & 17.4129 & 18.9629 & 20.3899 \\
\hline & 5 & 18.8962 & 19.1442 & 20.8872 & 18.4646 & 19.0172 & 20.9096 \\
\hline
\end{tabular}

TABLE 6: The running time to convergence for EMO, ABC, MQABC, FDE, FMQABC, and FMQABCA.

\begin{tabular}{|c|c|c|c|c|c|c|c|}
\hline \multirow{2}{*}{ Image } & \multirow{2}{*}{$M$} & \multicolumn{6}{|c|}{ Running time (second) } \\
\hline & & EMO & $\mathrm{ABC}$ & MQABC & FDE & FMQABC & FMQABCA \\
\hline \multirow{2}{*}{ Baboon } & 4 & 6.700095 & 2.176596 & 2.131156 & 3.665105 & 2.234492 & 2.983749 \\
\hline & 5 & 8.389706 & 2.269232 & 2.021364 & 5.288985 & 3.032174 & 3.766464 \\
\hline \multirow{2}{*}{ Lena } & 4 & 6.598210 & 2.202426 & 2.000834 & 3.847913 & 2.179925 & 3.307005 \\
\hline & 5 & 8.202104 & 2.275810 & 2.001621 & 5.351902 & 3.809324 & 4.252020 \\
\hline \multirow{2}{*}{ Aeroplane } & 4 & 7.877475 & 2.439630 & 2.318803 & 4.966922 & 2.562252 & 2.957771 \\
\hline & 5 & 9.311095 & 2.590907 & 2.448975 & 6.188636 & 3.045369 & 3.810145 \\
\hline \multirow{2}{*}{ Swan } & 4 & 7.778865 & 2.520282 & 2.305300 & 4.741355 & 2.194570 & 2.958491 \\
\hline & 5 & 9.368673 & 3.253198 & 3.045478 & 6.227627 & 3.575132 & 4.198971 \\
\hline \multirow{2}{*}{ Ladyhand } & 4 & 7.637196 & 2.582458 & 2.580046 & 4.742558 & 3.066803 & 3.515309 \\
\hline & 5 & 9.254623 & 3.219210 & 2.962865 & 6.399271 & 4.435080 & 5.078334 \\
\hline \multirow{2}{*}{ Starfish } & 4 & 7.722705 & 3.271955 & 3.013681 & 4.995923 & 2.806032 & 3.178964 \\
\hline & 5 & 9.123489 & 3.358983 & 3.075762 & 6.647341 & 4.417617 & 4.978053 \\
\hline \multirow{2}{*}{ Mountain } & 4 & 7.732155 & 2.408338 & 2.340408 & 4.723301 & 2.517399 & 3.096332 \\
\hline & 5 & 9.190040 & 3.063349 & 2.940541 & 6.345679 & 3.073803 & 4.989546 \\
\hline \multirow{2}{*}{ Building } & 4 & 7.612425 & 2.532897 & 2.452882 & 4.825658 & 2.951447 & 3.996561 \\
\hline & 5 & 9.296582 & 3.184240 & 2.981036 & 6.318346 & 3.586839 & 4.960509 \\
\hline
\end{tabular}



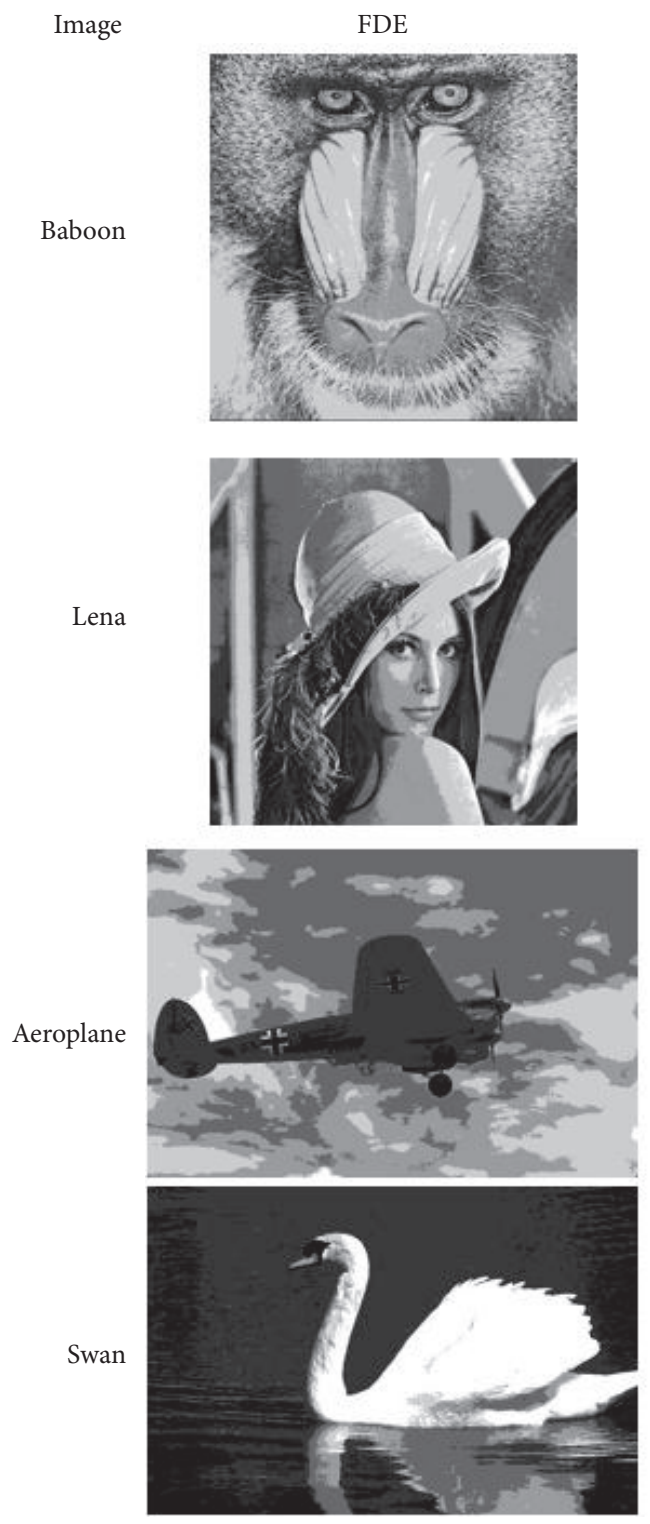

FMQABC
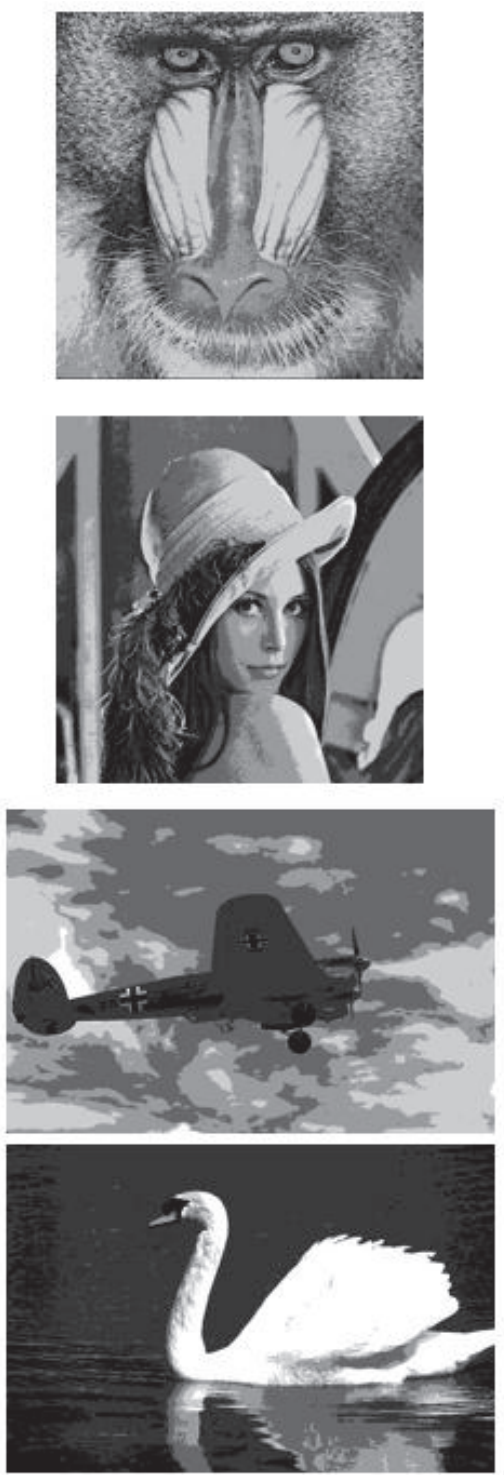

FMQABCA
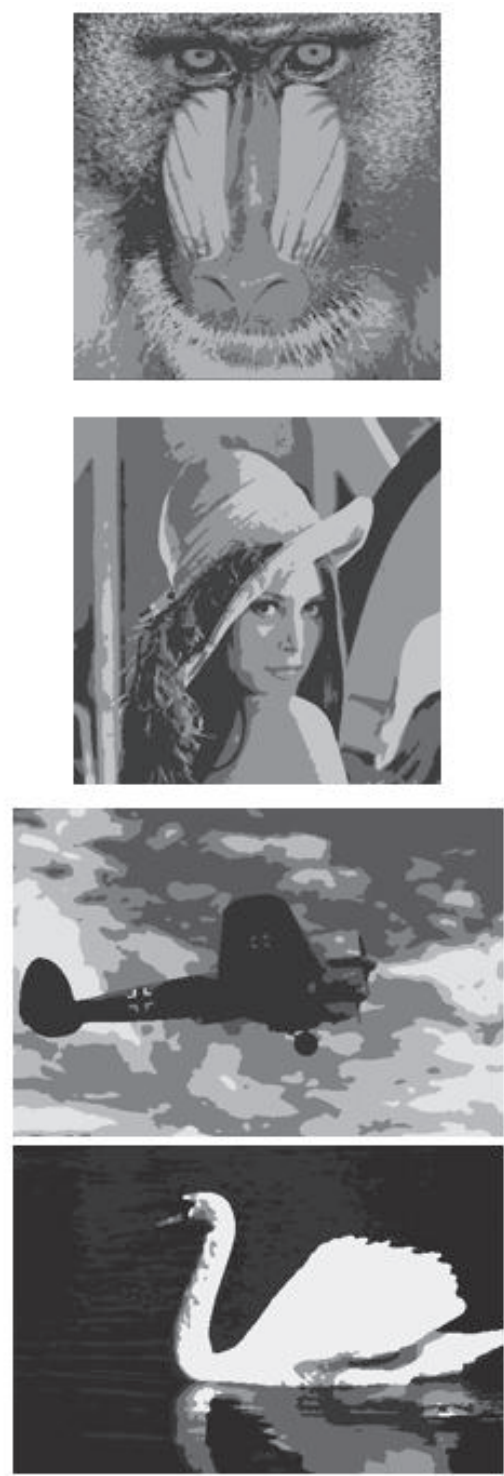

FIGURE 9: The comparison of grayscale segmentation results about (a)-(d) in Figure 1.

Education Institutions (PAPD), Jiangsu Collaborative Innovation Center on Atmospheric Environment, and Equipment Technology (CICAEET).

\section{References}

[1] S. Aja-Fernández, A. H. Curiale, and G. Vegas-Sánchez-Ferrero, "A local fuzzy thresholding methodology for multiregion image segmentation," Knowledge-Based Systems, vol. 83, no. 1, pp. 1-12, 2015.

[2] N. Shi and J. Pan, "An improved active contours model for image segmentation by level set method," Optik, vol. 127, no. 3, pp. 1037-1042, 2016.

[3] S. Masood, M. Sharif, A. Masood, M. Yasmin, and M. Raza, "A survey on medical image segmentation," Current Medical Imaging Reviews, vol. 11, no. 1, pp. 3-14, 2015.
[4] J. Torres-Sánchez, F. López-Granados, and J. M. Peña, "An automatic object-based method for optimal thresholding in UAV images: application for vegetation detection in herbaceous crops," Computers and Electronics in Agriculture, vol. 114, pp. 4352, 2015.

[5] M. Sonka, V. Hlavac, and R. Boyle, Image Processing, Analysis and Machine Vision, Springer US, Boston, Mass, USA, 1993.

[6] J. Li, X. Li, B. Yang, and X. Sun, "Segmentation-based image copy-move forgery detection scheme," IEEE Transactions on Information Forensics and Security, vol. 10, no. 3, pp. 507-518, 2015.

[7] D. Oliva, E. Cuevas, G. Pajares, D. Zaldivar, and V. Osuna, "A multilevel thresholding algorithm using electromagnetism optimization," Neurocomputing, vol. 139, pp. 357-381, 2014.

[8] B. Akay, "A study on particle swarm optimization and artificial bee colony algorithms for multilevel thresholding," Applied Soft Computing Journal, vol. 13, no. 6, pp. 3066-3091, 2013. 
[9] V. Osuna-Enciso, E. Cuevas, and H. Sossa, "A comparison of nature inspired algorithms for multi-threshold image segmentation," Expert Systems with Applications, vol. 40, no. 4, pp. 12131219, 2013.

[10] T. Kurban, P. Civicioglu, R. Kurban, and E. Besdok, "Comparison of evolutionary and swarm based computational techniques for multilevel color image thresholding," Applied Soft Computing Journal, vol. 23, pp. 128-143, 2014.

[11] S. Sarkar, S. Das, and S. S. Chaudhuri, "A multilevel color image thresholding scheme based on minimum cross entropy and differential evolution," Pattern Recognition Letters, vol. 54, pp. 27-35, 2015.

[12] J. N. Kapur, P. K. Sahoo, and A. K. C. Wong, "A new method for gray-level picture thresholding using the entropy of the histogram," Computer Vision, Graphics, and Image Processing, vol. 29, no. 3, pp. 273-285, 1985.

[13] N. Otsu, "Threshold selection method from gray-level histograms," IEEE Trans Syst Man Cybern, vol. 9, no. 1, pp. 62-66, 1979.

[14] X. Li, Z. Zhao, and H. D. Cheng, "Fuzzy entropy threshold approach to breast cancer detection," Information SciencesApplications, vol. 4, no. 1, pp. 49-56, 1995.

[15] J. Kittler and J. Illingworth, "Minimum error thresholding," Pattern Recognition, vol. 19, no. 1, pp. 41-47, 1986.

[16] M. Sezgin and B. Sankur, "Survey over image thresholding techniques and quantitative performance evaluation," Journal of Electronic Imaging, vol. 13, no. 1, pp. 146-168, 2004.

[17] I. Bloch, "Fuzzy sets for image processing and understanding," Fuzzy Sets and Systems, vol. 281, pp. 280-291, 2015.

[18] J. Anitha and J. D. Peter, "A spatial fuzzy based level set method for mammogram mass segmentation," in Proceedings of the IEEE 2nd International Conference on Electronics and Communication Systems (ICECS '15), pp. 1-6, 2015.

[19] C. I. Gonzalez, P. Melin, J. R. Castro et al., "Color image edge detection method based on interval type-2 fuzzy systems," in Design of Intelligent Systems Based on Fuzzy Logic, Neural Networks and Nature-Inspired Optimization, pp. 3-11, Springer International Publishing, 2015.

[20] F. Perez-Ornelas, O. Mendoza, P. Melin, J. R. Castro, A. Rodriguez-Diaz, and O. Castillo, "Fuzzy index to evaluate edge detection in digital images," PLoS ONE, vol. 10, no. 6, Article ID e0131161, 2015.

[21] Y. Zheng, B. Jeon, D. Xu, Q. M. J. Wu, and H. Zhang, "Image segmentation by generalized hierarchical fuzzy C-means algorithm," Journal of Intelligent and Fuzzy Systems, vol. 28, no. 2, pp. 961-973, 2015.

[22] H.-P. Chen, X.-J. Shen, and J.-W. Long, "Histogram-based colour image fuzzy clustering algorithm," Multimedia Tools and Applications, vol. 57, no. 18, pp. 11417-11432, 2015.

[23] F. Zhao, H. Liu, and J. Fan, "A multiobjective spatial fuzzy clustering algorithm for image segmentation," Applied Soft Computing, vol. 30, pp. 48-57, 2015.

[24] C. I. Gonzalez, P. Melin, J. R. Castro, O. Mendoza, and O. Castillo, "An improved sobel edge detection method based on generalized type-2 fuzzy logic," Soft Computing, vol. 20, no. 2, pp. 773-784, 2016.

[25] P. Ghamisi, M. S. Couceiro, F. M. L. Martins, and J. A. Benediktsson, "Multilevel image segmentation based on fractional-order Darwinian particle swarm optimization," IEEE Transactions on Geoscience and Remote Sensing, vol. 52, no. 5, pp. 2382-2394, 2014.
[26] A. K. Bhandari, A. Kumar, and G. K. Singh, "Modified artificial bee colony based computationally efficient multilevel thresholding for satellite image segmentation using Kapur's, Otsu and Tsallis functions," Expert Systems with Applications, vol. 42, no. 3, pp. 1573-1601, 2015.

[27] S. Sarkar, S. Paul, R. Burman et al., "A fuzzy entropy based multilevel image thresholding using differential evolution," in Swarm, Evolutionary, and Memetic Computing , pp. 386-395, Springer International Publishing, 2014.

[28] M. Zhao, A. M. N. Fu, and H. Yan, "A technique of threelevel thresholding based on probability partition and fuzzy 3partition," IEEE Transactions on Fuzzy Systems, vol. 9, no. 3, pp. 469-479, 2001.

[29] W.-B. Tao, J.-W. Tian, and J. Liu, "Image segmentation by three-level thresholding based on maximum fuzzy entropy and genetic algorithm," Pattern Recognition Letters, vol. 24, no. 16, pp. 3069-3078, 2003.

[30] G. Q. O. Pratamasunu, Z. Hu, A. Z. Arifin et al., "Image thresholding based on index of fuzziness and fuzzy similarity measure," in Proceedings of the IEEE 8th International Workshop on Computational Intelligence and Applications (IWCIA '15), pp. 161-166, IEEE, Hiroshima, Japan, November 2015.

[31] M. Muppidi, P. Rad, S. S. Agaian, and M. Jamshidi, "Image segmentation by multi-level thresholding based on fuzzy entropy and genetic algorithm in cloud," in Proceedings of the 10th System of Systems Engineering Conference (SoSE '15), pp. 143-148, IEEE, San Antonio, Tex, USA, May 2015.

[32] B. Akay and D. Karaboga, "A modified Artificial Bee Colony algorithm for real-parameter optimization," Information Sciences, vol. 192, pp. 120-142, 2012.

[33] D. Karaboga and B. Gorkemli, "A quick artificial bee colony -qABC- algorithm for optimization problems," in Proceedings of the International Symposium on INnovations in Intelligent SysTems and Applications (INISTA '12), 5, 1 pages, July 2012.

[34] S.-L. Jui, C. Lin, W. Xu, W. Lin, D. Wang, and K. Xiao, "Dynamic incorporation of wavelet filter in fuzzy C-means for efficient and noise-insensitive MR image segmentation," International Journal of Computational Intelligence Systems, vol. 8, no. 5, pp. 796-807, 2015.

[35] E. Cuevas, F. Sención, D. Zaldivar, M. Pérez-Cisneros, and H. Sossa, "A multi-threshold segmentation approach based on artificial bee colony optimization," Applied Intelligence, vol. 37, no. 3, pp. 321-336, 2012.

[36] P. Arbeláez, M. Maire, C. Fowlkes, and J. Malik, "Contour detection and hierarchical image segmentation," IEEE Transactions on Pattern Analysis and Machine Intelligence, vol. 33, no. 5, pp. 898-916, 2011. 


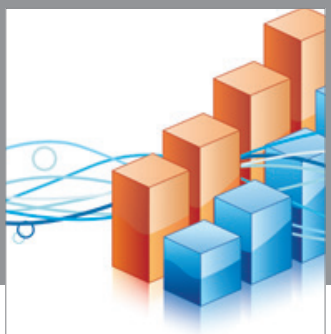

Advances in

Operations Research

vatem alat4

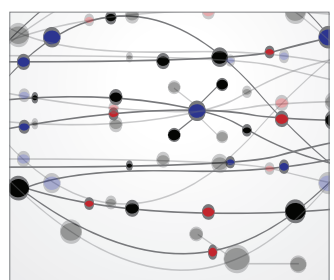

\section{The Scientific} World Journal
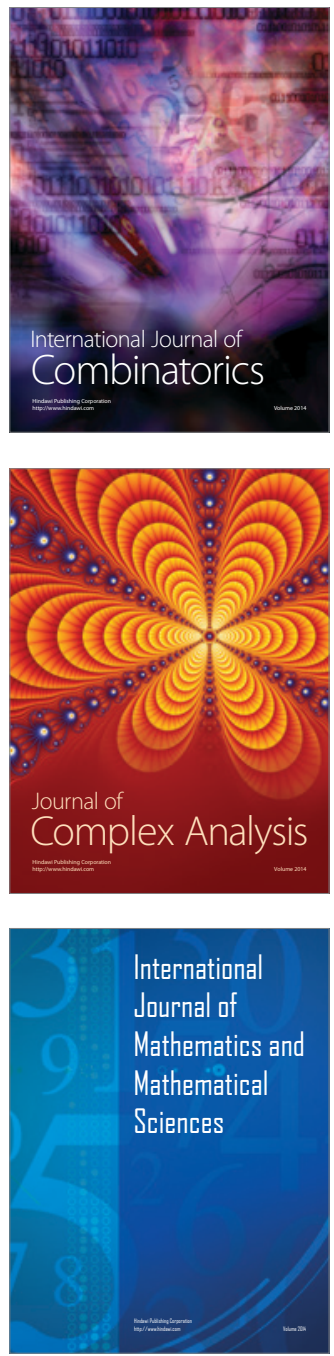
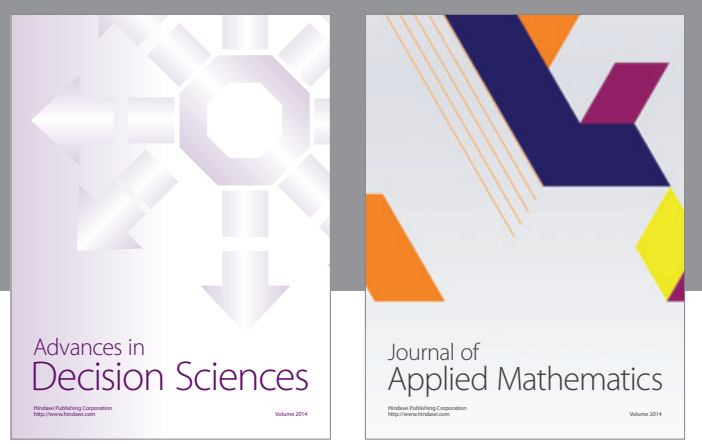

Algebra

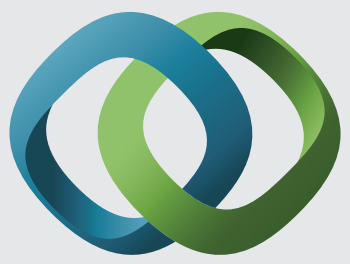

\section{Hindawi}

Submit your manuscripts at

http://www.hindawi.com
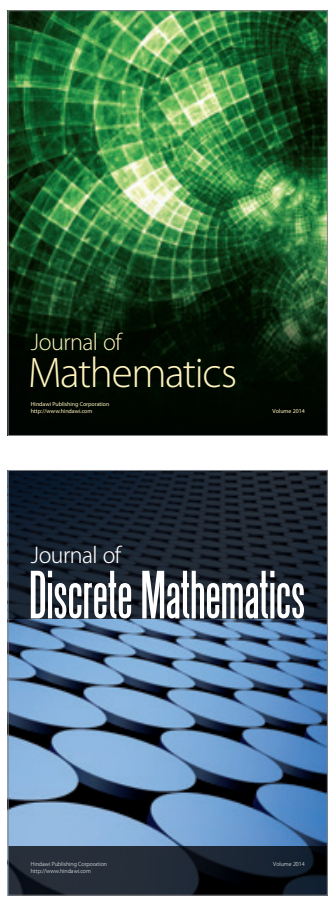

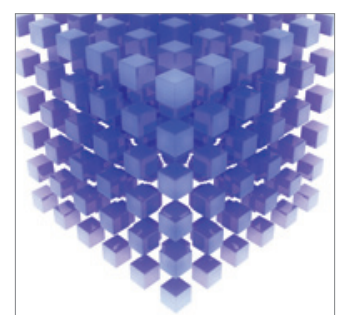

Mathematical Problems in Engineering
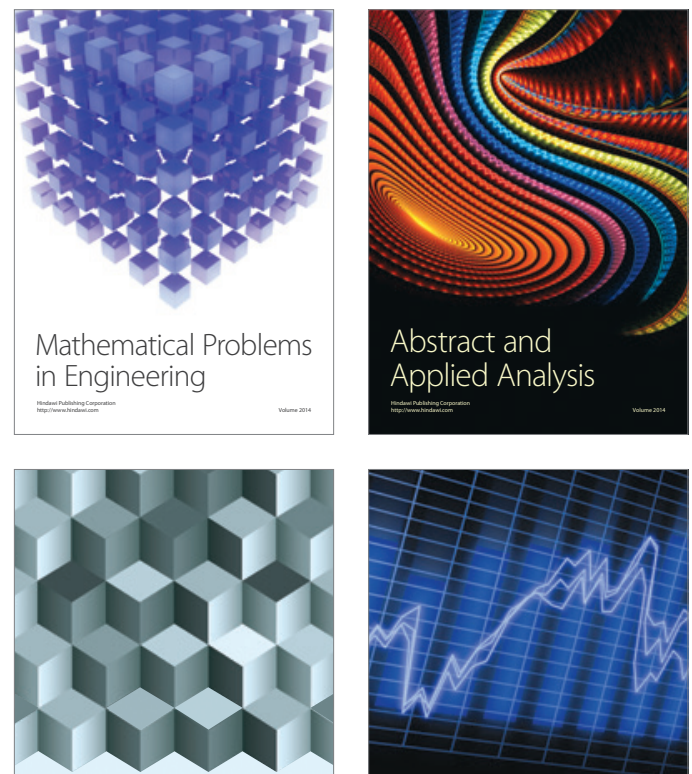

Journal of

Function Spaces

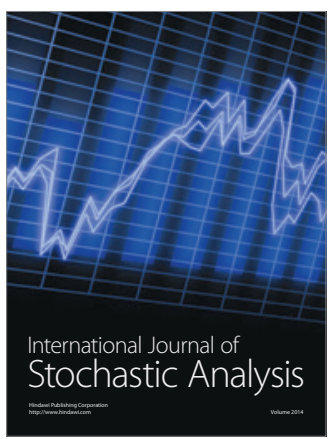

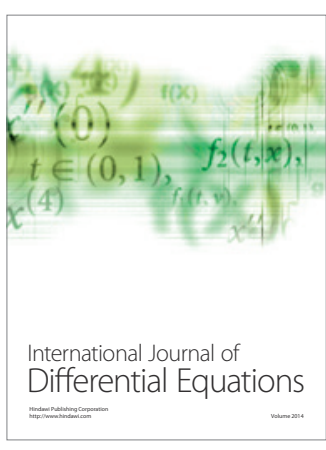
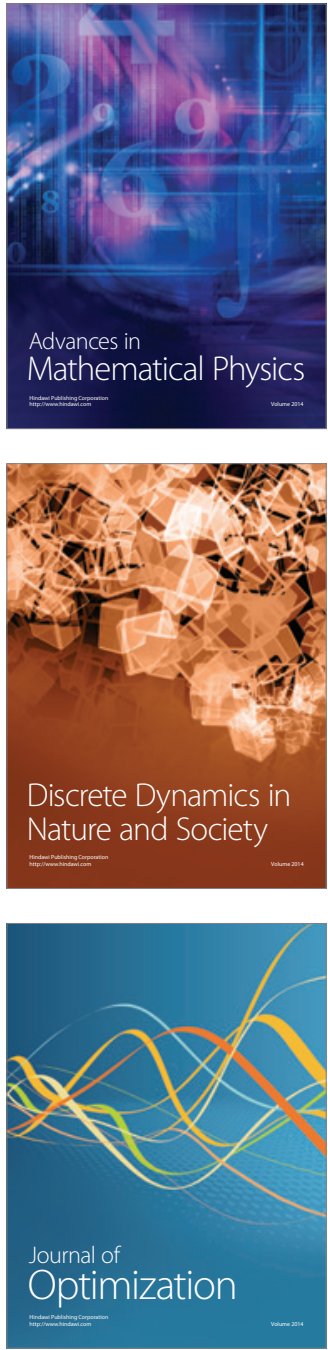